\title{
Vulnerability of adult hippocampus to toxicity after postnatal exposure of nursing male albino rats to iron overload : Histomorphometric and ultrastructural study
}

Original Article

\author{
Asmaa F. A. Dawood \\ Department of Histology, Faculty of Medicine, Assiut University, Assiut, Egypt, Department of \\ Biomedical Science, Faculty of Medicine, King Faisal University, Alhasa, Saudi Arabia
}

\begin{abstract}
Background: The association of iron overload with neurodegenerative diseases and cognitive deficits in aging were previous reported. Developing hippocampus is highly sensitive to changes in iron homeostasis that might lead to long term irreversible impact on its functions.

Objective: To examine the possible histopathological changes in adult hippocampal subregions CA1, CA3 and DG subjected to excess iron administration at critical early postnatal life.

Materials and Methods: 20 male rat pups weighing 50-55gm were selected and randomly divided into 2 groups (10 per each). The control received $0.5 \mathrm{ml}$ normal saline intraperitoneally once daily for 21 consecutive days. Iron treated group received intraperitoneal injection of ferrous sulfate dissolved in $0.5 \mathrm{ml}$ normal saline at a dose of $3 \mathrm{mg} / \mathrm{kg} / \mathrm{day}$ for 21 days. After sacrifice, brain specimens were processed for histomorphometric and ultrastructural study.

Results: Iron deposits were reported only in treated group using Prussian blue. Obvious degeneration of principal neurons with significant decrease in their number and increased immunoreactivity to caspase 3 and glial fibrillary acid protein (GFAP)were observed in all studied subfields of iron treated hippocampus. Astrogliosis, astrocyte damage and disruption of blood brain barrier were also noticed.

Conclusion: There was pronounced degenerative changes in all studied subregions of iron treated hippocampus indicating its extreme sensitivity to changes in iron homeostasis, hence iron overload could be considered highly toxic insult on growing hippocampal tissue.
\end{abstract}

Key Words: Astrogliosis, BBB, caspase 3, hippocampus, iron

Revised: 26 May 2019, Accepted: 26 June 2019

Corresponding Author: Asmaa F. A. Dawood, Department of Histology, Faculty of Medicine, Assiut University, Assiut, Egypt, Department of Biomedical Science, KFU, Alhasa, Saudi Arabia Tel.: +966568902355, E-mail: asmaanoorahmed@yahoo.com

ISSN:2536-9172, June 2019, Vol. 3, No. 1

\section{INTRODUCTION}

Hippocampus is a small curved formation in the brain. Its major subfields include hippocampus proper or cornu ammonis (CAl-4), dentate gyrus (DG) and subiculum. It is a key neural region that mediates higher brain functions as memory, spatial coding and is associated with learning, emotions and social behavior ${ }^{[1]}$. It has been reported that hippocampus is particularly sensitive to changes in homeostasis and is one of three brain regions that is most susceptible to oxidative stress and first to undergo decline in its function ${ }^{[2]}$.

Normal iron level (heme and non heme forms) is mandatory to support normal brain metabolic function, neurotransmission and for normal cognitive function and development. It plays a key role in myriads of physiological functions as oxygen transport, DNA synthesis, defense mechanisms, xenobiotic metabolism and myelin formation. It also contributes to maintain a necessary oxidative tone in neurons ${ }^{[3]}$. Unfortunately, these high requirements are coupled with high vulnerability of the brain to iron mediated oxidative damage. Escape of intracellular non heme iron $(\mathrm{Fe} 2+$ and $\mathrm{Fe} 3+)$ from normal sequestration with ferritin promotes reactive oxygen species (ROS) and oxidative stress damage ${ }^{[4]}$.

Prior efforts proved that iron and its major protein transferrin accumulate in different brain regions with aging causing memory and cognitive deficits ${ }^{[5]}$. Moreover, excess iron has been implicated in many human neurodegenerative diseases as Alzheimer's disease, Parkinson's disease, Huntington's disease. Iron overload is accused in neurodegeneration because of its participation in Fenton reaction and production of ROS which lead to neuronal damage $^{[6]}$.

Nowadays, routine iron fortification or supplementation is worldwide practiced during infancy. It is broadly recommended as a prophylactic measure against iron deficiency anemia, which commonly occurs in infancy especially breast-fed infants because of insufficient iron supply in breast milk. However, growing evidences reported iron overload as a consequence of iron fortification ${ }^{[7]}$. 
Ironoverload is also commonly seen among premature infants due to multiple erythrocyte transfusions and also among those with inherited defect in iron metabolism ${ }^{[8]}$. On the other hand, studies have reported adverse effects of iron fortified formula on infant and child linear growth and weight ${ }^{[9]}$. Moreover, recent concerns have been raised about whether the provision of iron to neonate and young children could lead to permanent drawbacks on brain functions ${ }^{[10]}$. Some efforts reported that neonatal iron administration could induce oxidative stress and memory deficits in rats $^{[11]}$ and human ${ }^{[12]}$. However, little is still known about such drawbacks on developing hippocampus. As a continuation for prior efforts, the current study aimed to figure out the potential long-term toxic effect of iron overload on the structure of hippocampal subfields CA1, CA3 and DG using neonatal rat model. As far as we know, it is the first detailed histomorphometric and ultrastructural analysis of excess iron overload on hippocampus being a major brain component involved in serious brain functions. We particularly select neonatal period as it is known as critical period which shows rapid brain growth ${ }^{[13]}$ coupled with high susceptibility to oxidative damage due to immature antioxidant system ${ }^{[14]}$.

\section{MATERIALS AND METHODS}

\section{Chemicals:}

-Ferrous sulfate heptahydrate $(\geq 99 \%)(\mathrm{FeSO} 47 \mathrm{H} 2 \mathrm{O})$ powder that was purchased from Sigma Aldrich, France. It was dissolved in normal saline and was given once daily by intraperitoneal injection at a dose of $3 \mathrm{mg} / \mathrm{kg} /$ day.

-Caspase 3 and GFAP antibodies were purchased from Thermo scientific Company-USA.

\section{Animals and experimental design:}

Pregnant albino rats were housed and kept under conventional housing conditions. They were purchased from Assiut University Animal Breeding Unit, Assiut, Egypt. Seven days after birth, 20 male pups weighing $50-55 \mathrm{gm}$ were selected and randomly divided into treatment and control groups (10 per each) as the following;

Control group: newborn rats received intraperitoneal injection of $0.5 \mathrm{ml}$ normal saline once daily for 21 consecutive days.

Iron treated group: animals received intraperitoneal injection of ferrous sulfate dissolved in $0.5 \mathrm{ml}$ normal saline at a dose of $3 \mathrm{mg} / \mathrm{kg} /$ day for 21 days $^{[15]}$.

Control and treated animals were kept separately and placed with their foster mothers. The rats had free access to food and water and humidity and temperature were kept constant. Care of the animals and the experimental procedures were done after approval and in accordance with ethical guidelines of ethical Committee, faculty of Medicine, Assiut University, Egypt.

At the end of the experiment, all animals were sacrificed under general anesthesia using chloroform. The skull vault was opened then brains were removed and immediately fixed and processed for light, electron and morphometric study.

\section{Methods: \\ Light microscopy:}

Brain specimens from temporal lobe were fixed in $10 \%$ neutral buffered formalin then processed for dehydration, clearing and embedding in melted paraffin. Coronal section of five $\mu \mathrm{m}$ paraffin sections were obtained and stained by hematoxylin and eosin (H\&E) for general histological study. For histochemical study, Prussian blue stain was used and counterstained with eosin stain for detection of ferric salts deposits in hippocampal tissue. Ferric salts precipitate appears as small blue particles or deposits ${ }^{[16]}$.

\section{Immunohistochemistry:}

A series of paraffin sections was immunolabeled for caspase 3 as indicator of apoptosis and for GFAP as a marker of astrocytes. $5 \mu \mathrm{m}$ paraffin sections were cut and placed in charge glass slides then dewaxed in xylene and rehydrated using descending grades of alcohol. Then sections were incubated in $10 \%$ hydrogen peroxide for 10 minutes to block endogenous peroxidase activity then slides were washed in phosphate buffered saline (PBS) at $\mathrm{pH}=7.4$ for $5 \mathrm{~min}$. To unmask the antigenic sites, sections were put into citrate buffer $0.01 \mathrm{~mol} / 1(\mathrm{pH}=6)$ in a microwave for 10 min. Then sections were incubated in $1 \%$ bovine serum albumin dissolved in PBS at $370 \mathrm{C}$ for 30 min to prevent the nonspecific background staining. Incubation was done with the following primary antibodies (anti-caspase 3; rabbitcaspase 3 polyclonal antibodyand anti-glial fibrillary acid protein;anti-GFAP; Ab-1, mouse monoclonal antibody, GA-5) at a dilution of (1/100) for one hour at room temperature. The sections were rinsed with PBS, and then incubated with biotinylated secondary antibodies. Finally, the sections were incubated in 3,3-diaminobenzidene (DAB) for $15 \mathrm{~min}$ and were counterstained by Mayer's hematoxylin, then dehydrated, cleared and mounted. Negative control sections were prepared using PBS without using primary antibody. GFAP immunostained astrocytes appeared as small brownish branching cells and caspase 3 positive cells exhibited dark brown cytoplasmic granules and their nuclei stained blue ${ }^{[17]}$.

\section{Transmission electron microscopy:}

After dissection, small specimens of hippocampus were fixed in $4 \%$ cold glutaraldehyde in cacodylate buffer (pH 7.4) for $24 \mathrm{~h}$ then post fixed in osmium tetroxide (1\%) in phosphate buffer for $2 \mathrm{~h}$. Specimens were dehydrated in ascending grades of alcohol, cleared with propylene oxide, 
then embedded in Epon-812 substitute. Using ultratome, Semi-thin sections $(0.5 \mu \mathrm{m})$ were cut with glass knives, stained with $1 \%$ toluidine blue $(\mathrm{pH} 7.3)$ and examine with light microscope. Ultrathin sections $(50 \mathrm{~nm})$ from CA1, CA3 and DG regions were selected and cut on a Reichert ultratome, mounted on copper grids, and contrasted with uranyl acetate and lead citrate $^{[18]}$. These sections were examined and photographed using the TEM (Jeol EM-100 CX11; Japanese electron optic laboratory, Tokyo, Japan)in Assiut UniversityElectron Microscope Unit, Assiut, Egypt.

\section{Morphometric and statistical study:}

Quantitative morphometric measurement was done by using soft image analyzer computer system in the department of histology, Faculty of medicine, Assiut University, Egypt. 1n H\&E stained sections; five nonoverlapping fields of five serial sections of five animals were used. The number of dark (degenerated) cells and light (normal) pyramidal and granule cells (from limb region) in CA1, CA3 and DG of studied groups were counted by touch count method using lens $\times 400^{[19]}$.

Statistical analysis of results was done using SPSS for windows Version 16; SPSS Inc., Chicago, IL, USA. Data analysis was done using Mann-Whitney test to compare means among groups. Results were expressed as mean \pm SD. $P$ value of less than 0.05 was considered statistically significant.

\section{RESULTS}

\section{Light microscope examination:}

\section{[1] $\mathrm{H} \& \mathrm{E}$ stained sections:}

In H\&E stained sections, the major parts of control hippocampus includedhippocampus proper or Ammon's horn (Cornu Ammonis "CA"), dentate gyrus and subiculum. The $\mathrm{C}$ shaped Ammon's horn was interdigitating with $\mathrm{V}$ or U-shaped dentate gyrus. Ammon's horn was formed of CA1, CA2, CA3 and CA4 regions. CA2 was a small transitional area interposed between $\mathrm{CA} 1$ and $\mathrm{CA} 3$. CA4 represented the terminal portion that projected and lied within the concavity of the dentate gyrus. Subiculum appeared as outward continuation of CA1. CA1 was separated from dentate gyrus by hippocampal fissure or sulcus (Fig. 1).

H\&E stained sections of iron treated rats showed obvious degenerative changes of all studied hippocampal subregions mainly in CA1 and DG and apparently to less extent in CA3. The changes were mostly patchy with some areas seemed more affected than others (Fig. 2).

In the control group, CA1 region was formed of four layers arranged from deep to superficial as; stratum oriens, stratum pyramidale, stratum radiatum and stratum lacunosum moleculare. The pyramidal layer or stratum pyramidale is the principal layer that harbored the soma (perikarya) of pyramidal neurons. CAl pyramidal cells were small and were arranged as closely packed superficial layer and more loosely organized deep layer. They appeared triangular and had large rounded vesicular nuclei with prominent nucleoli and scanty basophilic cytoplasm. Their apical and basal dendrites were projecting towards stratum radiatum and oriens, respectively. Supporting glial cells and blood vessels were scattered in different strata. Astrocytes exhibited rounded vesicular nuclei, oligodendrocytes had small dense nuclei and perinuclear halos and microglia had rod shaped nuclei (Fig. 3a).CA3 subfield of the control group was composed of the same layers as CA1 in addition to stratum lucidum, which appeared as very narrow zone between stratum pyramidale and stratum radiatum. Pyramidal neurons of CA3 exhibited the same appearance as those of CA1 but appeared larger and more loosely arranged (Fig. 4a).

In $\mathrm{H} \& \mathrm{E}$ stained sections of both $\mathrm{CA} 1$ and $\mathrm{CA} 3$ regions of iron treated hippocampus, pyramidal neurons were affected, however, degenerative changes were apparently more frequent and more obvious in CA1 than CA3. Pyramidal neurons apparently decreased in their number. The remaining neurons were mostly degenerated, disorganized and exhibited irregular outlines with darkly stained cytoplasm and dense nuclei. Empty spaces or clear zones were noticed around degenerated neurons due shrinkage of their soma. Glial cells appeared more frequent than the control group indicating gliosis (Figs. $3 b$ and $4 b$ ).

H\&E section of dentate gyrus of control group showed its three layers including stratum moleculare, stratum granulosum and polymorphic layer. The granule and molecular layers formed a V or U-shaped structure "fascia dentata", which was composed of ectal (upper) and endal (lower) limbs or blades connected by the crest. Fascia dentata enclosed the hilus, the later included the polymorphic layer of dentate gyrus and pyramidal layer of CA4 (Figs. 1 and 5a). Granule cell layer is the principal cell layer of dentate gyrus. It contained the perikarya of the granule cells, which were arranged into several tightly packed strata. They were small rounded cells having scanty basophilic cytoplasm and rounded pale large vesicular nuclei. Some granule cells with oval dense nuclei and dark cytoplasm were found in the subgranule zone, wedged between granule cell and polymorphic layers. Polymorphic layer harbored large pyramidal neurons. Glial cells and blood vessels were observed (Fig. 5a).

In H\&E sections in DG of iron treated group, the granule cell layer apparently appeared thinner and less in number of cells than the control group. It contained numerous degenerated cells with irregular outlines and surrounded by numerous glial cells (gliosis). They had densely stained cytoplasm and nuclei. Focal neuronal loss was also observed (Fig.5b). 


\section{[2] Semithin stained sections:}

In semithin sections of control CA1, pyramidal neurons had large rounded pale vesicular nuclei with prominent nucleoli that occupying most cytoplasm. They were supported by scattered population of astrocytes. Astrocytes had pale ovoid nuclei, lightly stained cytoplasm with some cytoplasmic processes and could be seen near the vicinity of blood vessels (Fig. 6a). Semithin sections of CA3 of the control group showed large branching pyramidal neurons that exhibited similar appearance as CA1 neurons and were supported with astrocytes and blood vessels (Fig. 7a).

Semithin sections of both CA1 and CA3 of iron treated group showed degeneration, shrinkage and disorganization of many neurons. Many reactive astrocytes were noticed with pyknotic nuclei and vacuolated cytoplasm indicating astrogliosis (Figs. 6b and 7b)

Examination of toluidine blue stained semithin of DG of the control group showed rounded closely packed granule cells with large central pale rounded or oval nuclei and prominent eccentric nucleoli and were surrounded by thin rim of cytoplasm. Their apical dendritic processes were projecting toward stratum moleculare. Astrocytes and blood vessels were also observed (Fig. 8a).

In semithin sections of DG of iron treated group, many granule cells exhibited degeneration and shrinkage similar to those noticed in other studied areas of treated hippocampus (Fig. 8b).

\section{[3] Histochemical results (Prussian blue stained sections):}

Examination of Prussian blue stained sections of all studied subfields ofcontrol hippocampus (CA1, CA3 $\& D G)$ revealed absence or negative reaction for ferric ion particles in all strata (Figs. 9a,10a and11a).

In contrast to control group, positive iron reaction in the form of blue deposits or precipitate was detected in all strata of studied subregions of iron treated hippocampus. The reaction apparentlyappearsmore pronouncedin DG than in CA1 and CA3 (Figs. 9b, 10b\&11b)

\section{[4] Immunohistochemical results:}

\section{Caspase 3 immunostainiing:}

Immunohistochemical stain for demonstration of Caspase 3 showed very weak or almost negative reaction in the cytoplasm of pyramidal cells in different regions of control hippocampus (CA1, CA3 and DG) (Figs. 12a, 13a and 14a).

Caspase 3 expression showed strong positive reaction in pyramidal cells of studied subregions of iron treated hippocampus (CA1, CA3 and DG). The positive reaction appeared as dark brownish granules staining the cytoplasm of many perikarya (Figs. 12b, 13b and 14b).

\section{GFAP immunostaining:}

In all studied fields of the control hippocampus, GFAP immunostained section showed some positively stained astrocytes appeared as small scattered brownish satellite shaped cells. They exhibited small cell body with long thin branching processes seen in all layers and extending among neurons and near blood capillaries (Figs. 15a and 16a).

In contrast to the control group, all studied CA-DG fields of iron treated hippocampus exhibited increased frequency of positively stained astrocytes that had profound branching processes (Figs. 15b and 16b).

\section{Electron microscope examination:}

Ultrathin sections of control CA1 pyramidal neurons showed typical morphology of perikaryon including large central oval or rounded euchromatic nucleus with prominent nucleolus and intact nuclear envelope. Its cytoplasm was rich in many organelles mainly rER, free ribosomes, intact mitochondria and some multivesicular bodies (Figs. 17 and 18).

Neurons in different hippocampal regions of iron treated group showed obvious histopathological alternations compared with the control group.In ultrathin sections of pyramidal neurons of CA1 treated group, many neurons appeared degenerated with variable density. They were surrounded by large empty spaces indicating lysis of the adjacent neuropil and retraction of their soma. Paler degenerated neurons exhibited pale rarified cytoplasm, which lost most organelles leaving large empty cytoplasmic spaces. Still, few partially damaged mitochondria were noticed. Their nuclei appeared euchromatic with indented outline. Dark degenerated neurons were smaller and shrunken with irregular outline. Their cytoplasm was electron dense with poorly defined organelles except for numerous lysosomes and some rER cisternae. Their nuclei were shrunken with irregular outline (Fig. 19).

Ultrastructural examination of control CA3 pyramidal neurons revealed similar subcellular morphology to CA1 neurons but CA 3 neurons were larger. Their cytoplasm was abundant and rich in organelles as rER, free ribosomes, mitochondria, and Golgi apparatus, with some lysosomes and multivesicular bodies. The nucleus was rounded euchromatic with prominent nucleolus and had intact nuclear envelope (Fig. 20).

Ultrathin section of CA3 pyramidal neurons of iron treated group showed similar subcellular degenerative changes as those noticed in CA1 neurons. Paler neurons exhibited ill-defined cell membrane. Their cytoplasm was rarified with obvious damage of most organelles. Mitochondria with partially damaged cristae were observed. Its nucleus was euchromatic with indented outline. Dark shrunken degenerated neurons exhibited irregular outline, small shrunken irregular nucleus and 
electron dense cytoplasm containing many lysosomes and some rER cisternae (Fig. 21).

In ultrathin sections of granule cells of control DG, their nuclei appeared oval or rounded and euchromatic with prominent nucleoli. Cytoplasm contained some cisternae of rER, ribosomes and mitochondria (Fig. 22).

Ultrastructural examination of granule cells in DG of treated group showing similar degeneration of their perikarya and the surrounding neuropil as neurons in other regions of treated hippocampus. Pale degenerating neurons had lost some organelles leaving empty spaces. However, some organelles were still observed as free ribosomes, deformed mitochondria, Golgi apparatus associated with vesicular structure, rER cisternae and lysosomes. Dark markedly shrunken neurons were observed with apoptotic nuclei and dense cytoplasm containing some organelles (Fig. 23).

In different regions of control hippocampus, astrocytes exhibited its typical and characteristic ultrastructural appearance and could be noticed adjacent to neurons. Its nucleus was oval, euchromatic and was delineated by thin rim of heterochromatin. Its cytoplasm was scanty, pale or electron lucent and contained few organelles as mitochondria (Figs. 17, 22a and 24a).

Ultrastructural examination of astrocytes in all studied regions of treated hippocampus showed reactive astrogliosis indicated by markedly swollen electron lucent cytoplasm that contained few damaged mitochondria, dilated rER and free ribosomes (Figs. 23a and 24b).

Blood capillaries of the control group were lined by squamous endothelial cells that had flattened nuclei containing heterochromatin clumps and its cytoplasm had few organelles. Their lumen harbored erythrocytes and their wall were surrounded by thin regular basal lamina and supported by pale electron lucent astrocyte processes or end feet (Fig. 25a).

In iron treated group, blood capillaries revealed irregular and thickened basal lamina. Their lumen was irregular and contained deformed erythrocytes. Their wall was surrounded by extremely swollen electron lucent astrocyte processes or end feet that harbored few swollen and damaged mitochondria (Fig. 25b).

\section{Morphometric results:}

Statistical analysis of all studied subfields of iron treated hippocampus (CA1, CA2 and CA3) showed significant increase in the mean count of dark (degenerated) principal neurons (pyramidal and granule cells) per field when compared with the control group (Table 1 and Figure 1').

In contrast, the mean number of light (normal) cells/ field was significantly decreased in all subregions of treated hippocampus (CA1, CA3 and DG) compared with control group (Table 2 and Figure 2').

Table 1: The mean \pm SD of dark (degenerated) cell count/field in CA1, CA3 and DG regions of control and iron treated hippocampus

\begin{tabular}{|c|c|c|c|}
\hline \multirow{2}{*}{ Group name } & Control & Treated & $P$-value \\
\hline & Mean \pm SD & Mean \pm SD & \\
\hline \multirow[t]{2}{*}{ CA1 } & $1.04 \pm 0.889$ & $14.52 \pm 4.417$ & \\
\hline & Range (0-3) & Range (10-26) & \\
\hline \multirow[t]{2}{*}{ CA3 } & $1.04 \pm 935$ & $13.60 \pm 2.915$ & $0.000^{* * *}$ \\
\hline & Range (0-3) & Range (6-17) & \\
\hline \multirow[t]{2}{*}{ DG } & $1.28 \pm 0.980$ & $20.16 \pm 4.089$ & \\
\hline & Range (0-3) & Range (14-29) & \\
\hline
\end{tabular}

* Statistical significant difference $(P<0.05)$

Ctr CA1vs.T CA1: $0.000^{* * *}$

Ctr CA3vs.T CA3: $0.000 * * *$

Ctr DGvs.T DG: $0.000^{* * *}$

(Mann-Whitney Test) 


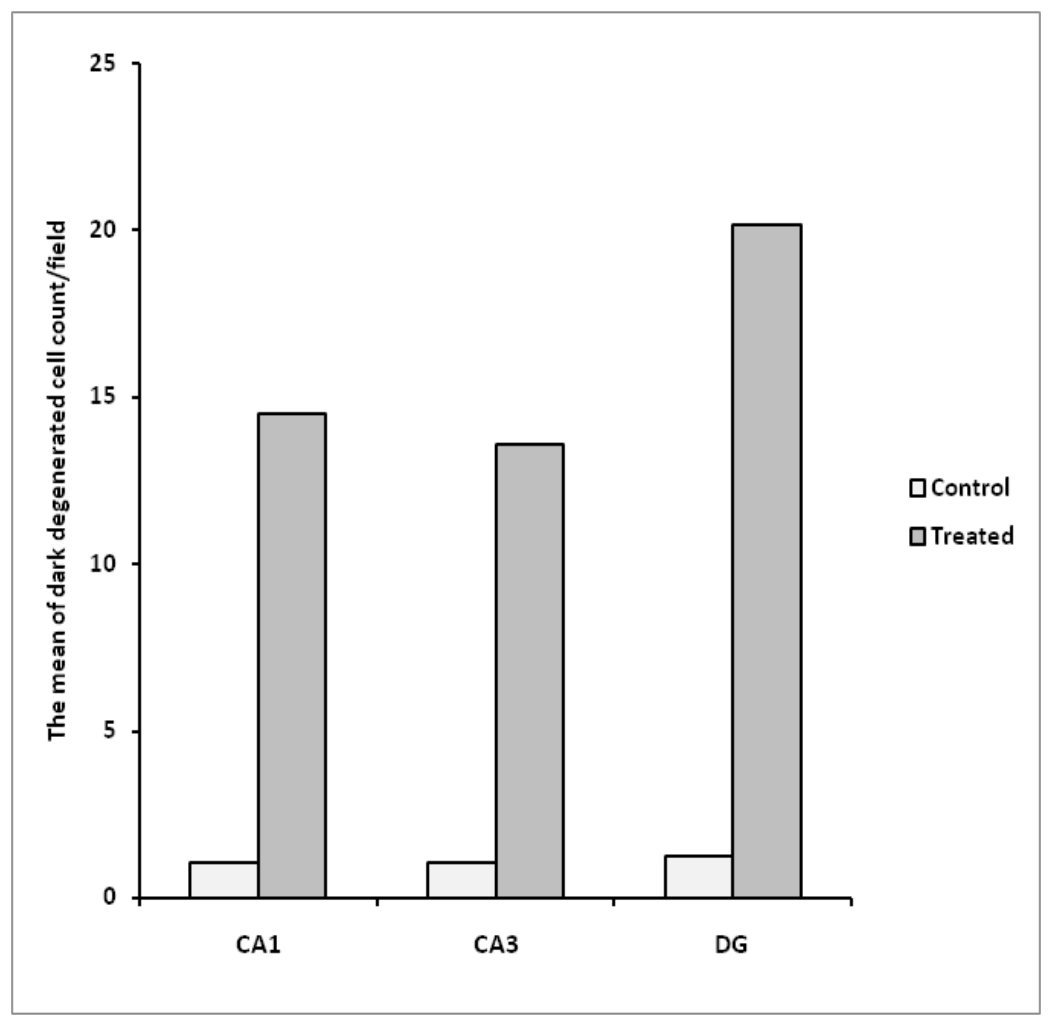

Fig. 1': The mean \pm SD of dark degenerated cell count/ field in CA1, CA3 and DG regions of control and iron treated hippocampus

Table 2: The mean \pm SD of light (normal) cell count/field in CA1, CA3 and DG regions of control and iron treated hippocampus

\begin{tabular}{|c|c|c|c|}
\hline \multirow{2}{*}{ Group name } & Control & Treated & \multirow[t]{2}{*}{$P$-value } \\
\hline & Mean \pm SD & Mean \pm SD & \\
\hline \multirow[t]{2}{*}{ CA1 } & $71.04 \pm 3.102$ & $23.48 \pm 6.462$ & \\
\hline & Range (66-77) & Range (10-33) & \\
\hline \multirow[t]{2}{*}{ CA3 } & $41.92 \pm 4.663$ & $19.96 \pm 3.048$ & $0.000^{* * *}$ \\
\hline & Range (35-50) & Range (15-26) & \\
\hline \multirow[t]{2}{*}{ DG } & $113.20 \pm 5.664$ & $39.64 \pm 4.618$ & \\
\hline & Range (100-120) & Range (30-54) & \\
\hline
\end{tabular}

* Statistical significant difference $(P<0.05)$

Ctr CA1vs.T CA1: $0.000^{* * *}$

Ctr CA3vs.T CA3: $0.000^{* * *}$

Ctr DGvs.T DG: $0.000^{* * *}$

(Mann-Whitney Test) 


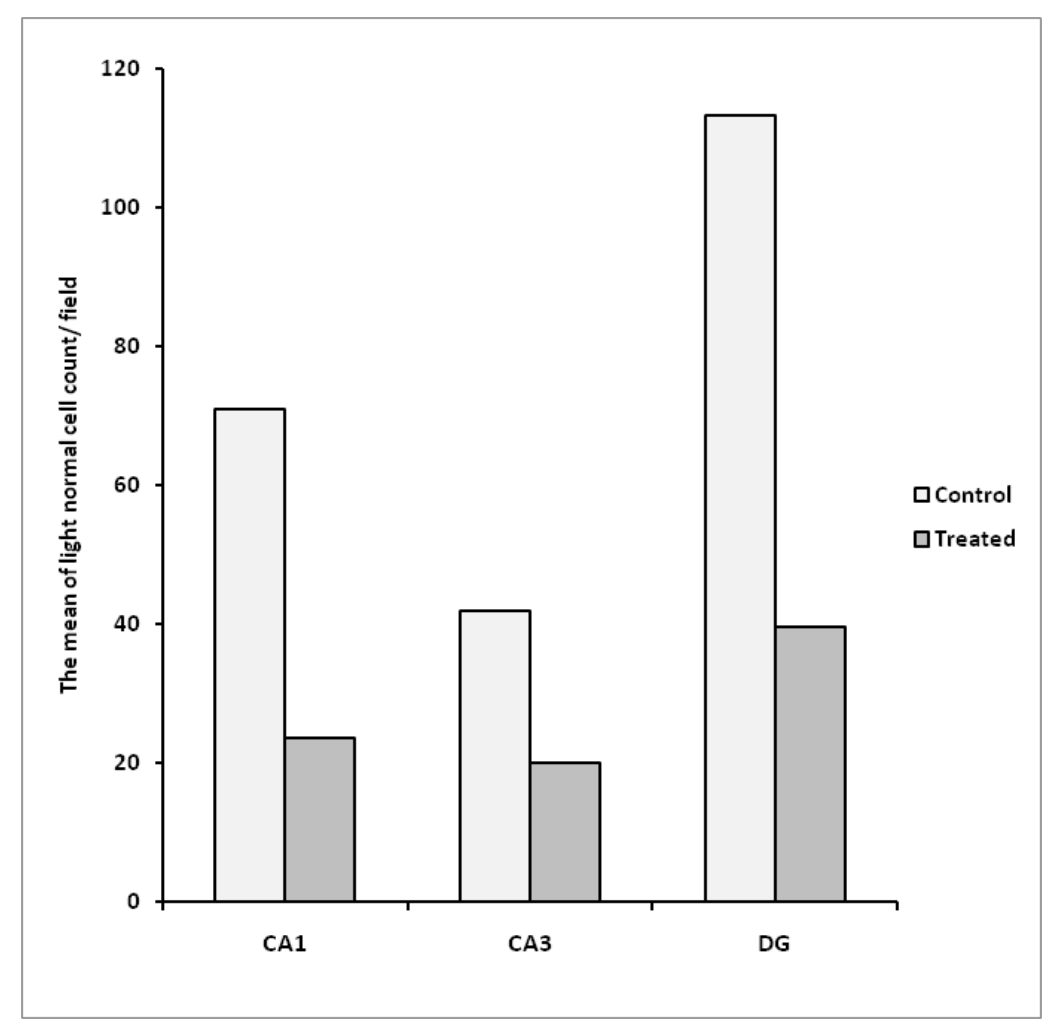

Fig. 2': The mean \pm SD of light normal cell count/ field in CA1, CA3 and DG regions of control and treated hippocampus

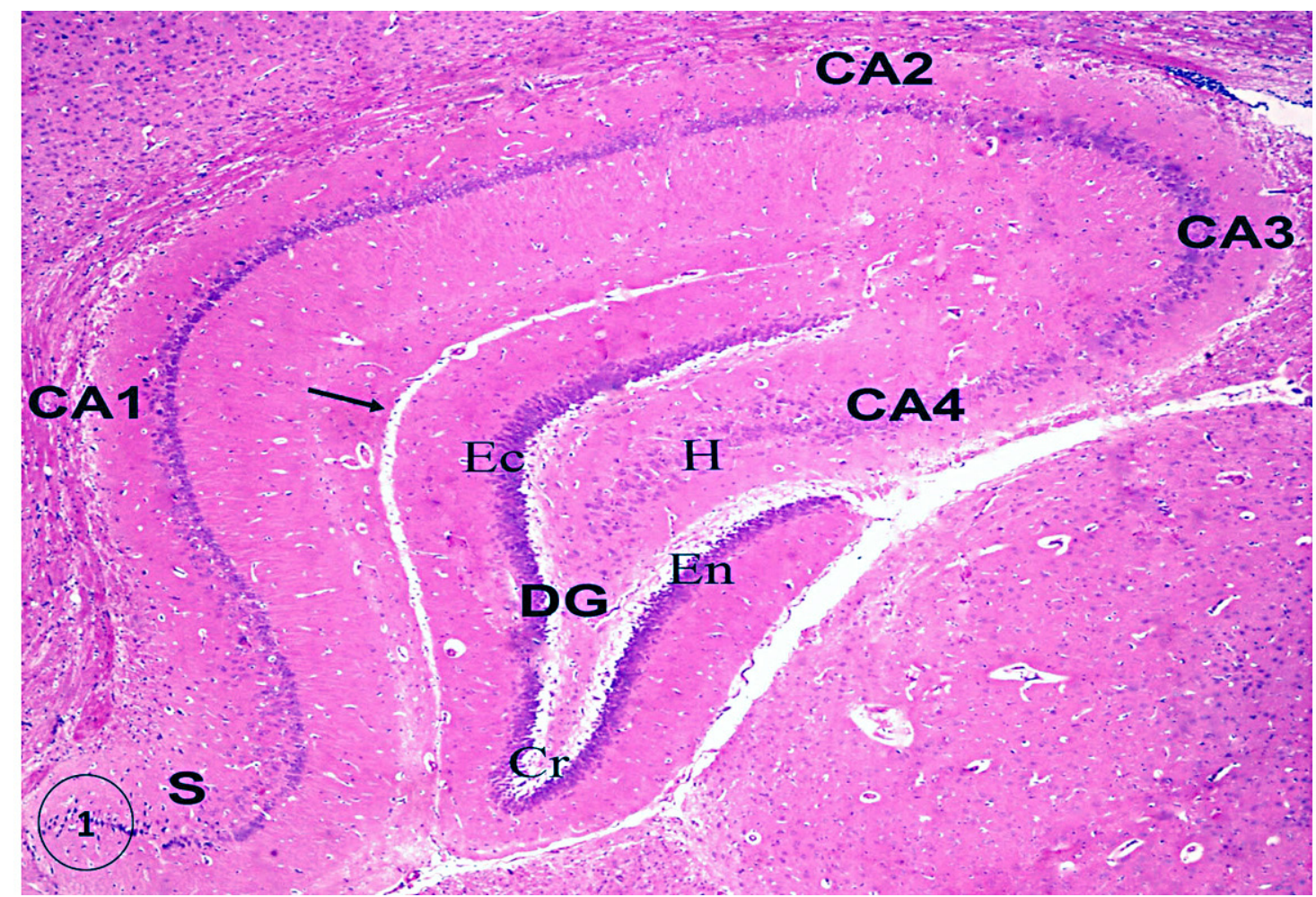

Fig. 1: A photomicrograph of a section in control hippocampus showing Ammon's horn CA1, CA2, CA3 and CA4 regions, dentate gyrus (DG) and subiculum (S). Dentate gyrus has ectal (Ec) and endal (En) limbs, crest (Cr) and hilus (H). Subiculum is continuous with Ammon's horn at CA1. Notice; hippocampal fissure separating CA1 from DG (arrow) (H\&E x 40). 


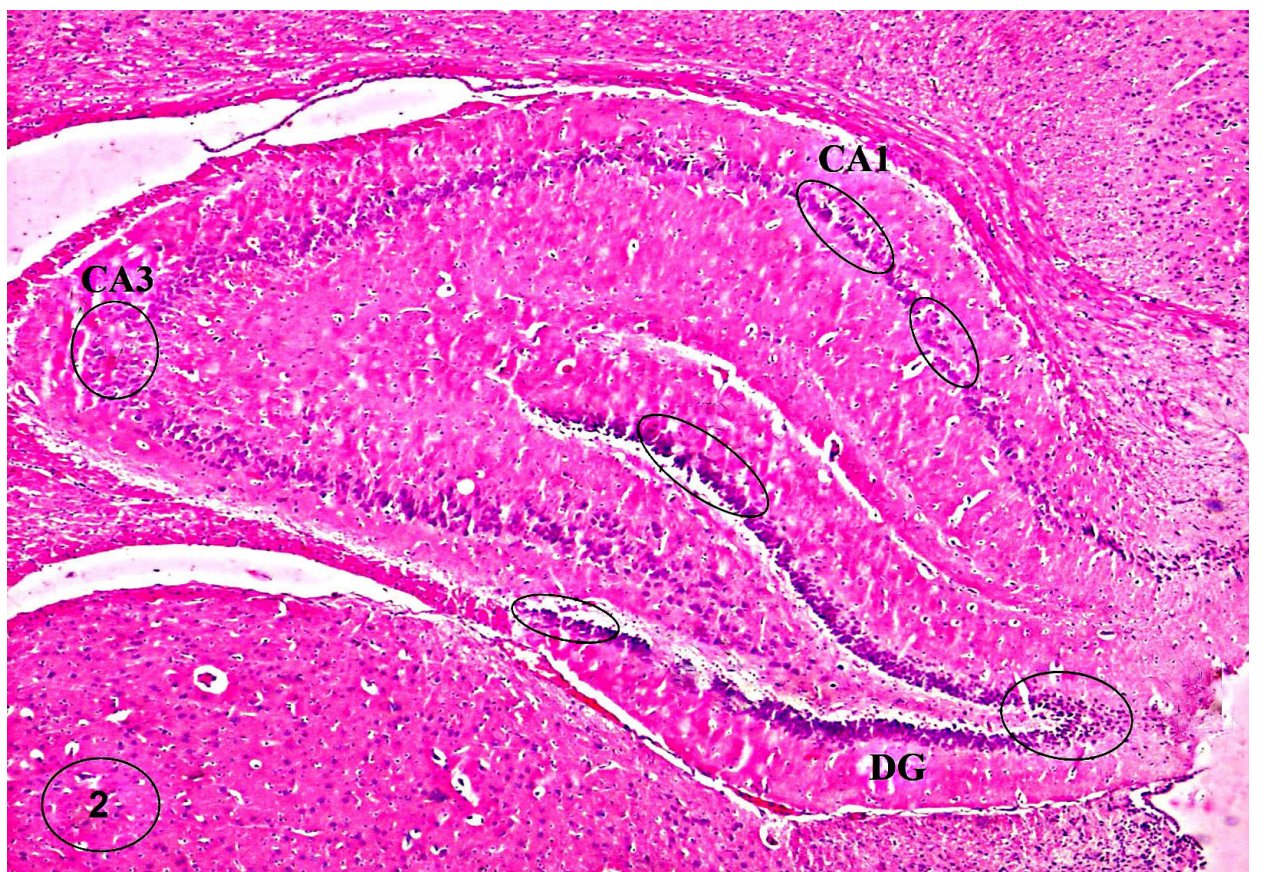

Fig. 2: A photomicrograph of a section in the hippocampus of iron treated group showing remarkable degenerative changes affecting all studied regions of hippocampus being apparently more frequent and more obvious in CA1 and DG than CA3. These changes tend to be patchy with some regions appear more affected than others $(\bigcirc)(\mathrm{H} \& \mathrm{E} x 40)$.
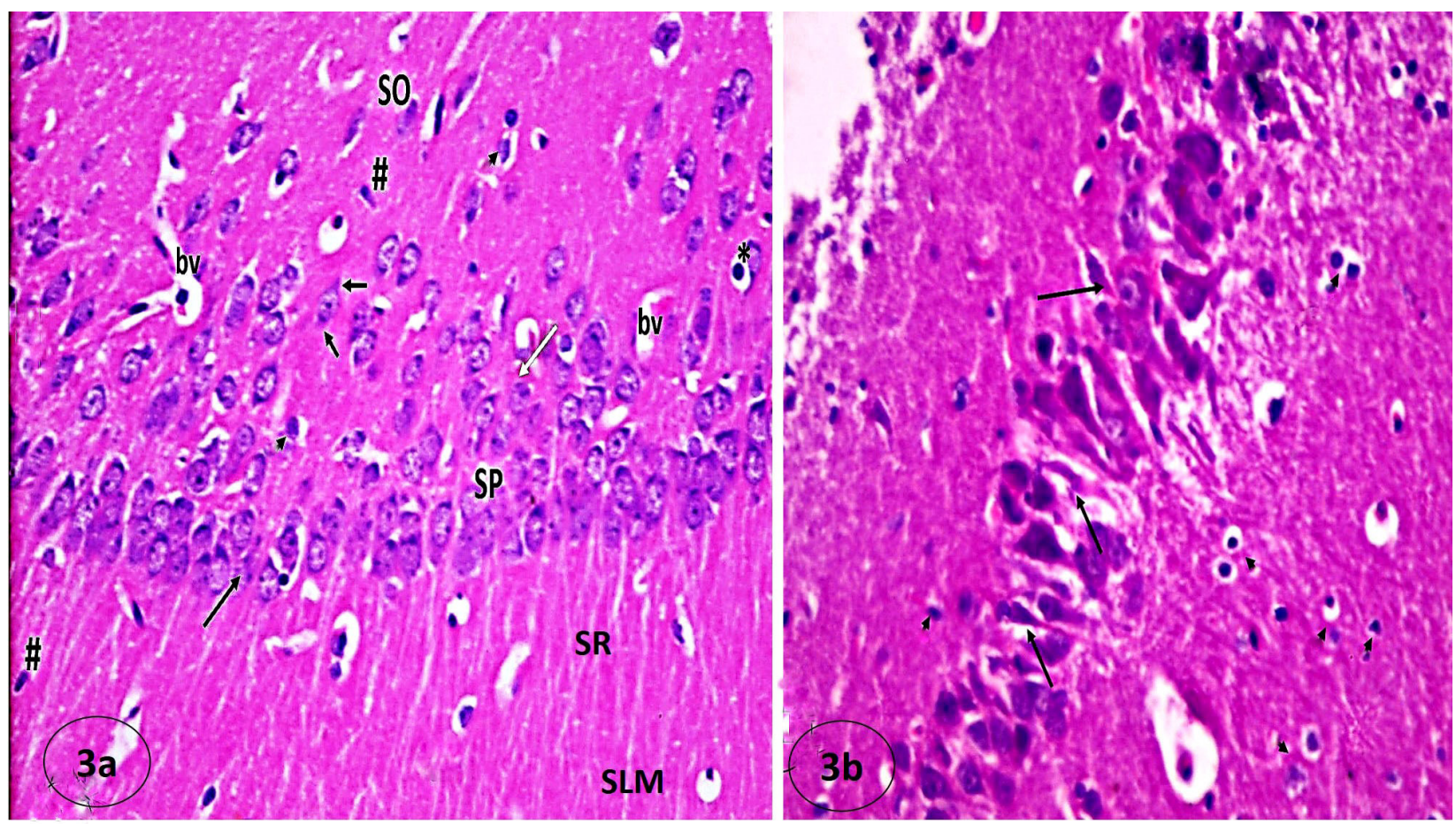

Fig. 3: Photomicrographsof CA1 subregion (3a) CA1 of the control group showing its strata; oriens (SO), pyramidale (SP), radiatum (SR) and lacunosummoleculare (SLM). Small triangular pyramidal neurons are arranged as loosely packed deep layer (white arrow) and tightly packed superficial layer (black arrow). They have apical and basal processes (short arrows) that projecting towards SR and SO respectively.Notice, blood vessels (bv) and different glial cells including astrocytes with pale rounded nuclei (arrow heads), oligodendrocyte with dark nuclei surrounded by perinuclear halos $(*)$ and microglia with rod shaped nuclei (\#). (3b) CA1 of iron treated group showing most pyramidal cells are disorganized and degenerated with irregular outline and are surrounded by empty spaces (arrows). They have deeply stained cytoplasm and dense nuclei. There are many glial cells compared with the control indicating gliosis (arrow heads) (H\&E X400). 

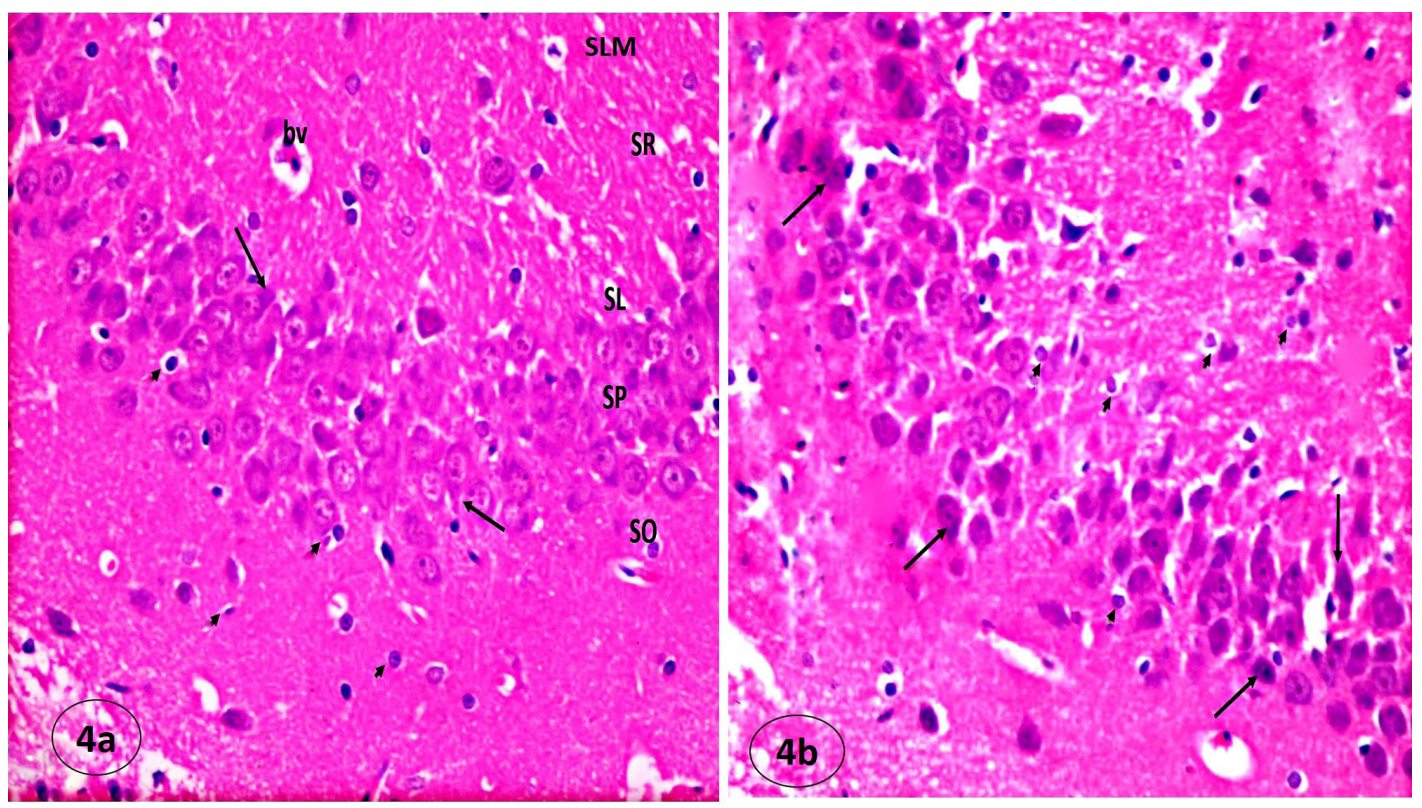

Fig. 4: Photomicrographs of CA3 subregion (4a) CA3 of the control group showing its strata; oriens (SO), pyramidale (SP), lucidum (SL), radiatum (SR) and lacunosummoleculare (SLM). Its pyramidal cells appear larger in size and more loosely packed thanCA1neurons. They are supported by glial cells (arrow heads) and are supplied by blood vessels (bv). Their cytoplasmic projections are directed towards SR and $\mathrm{SO}$ as seen in CA1 (arrows). (4b) CA3 of iron treated group showing some degenerated pyramidal cells having irregular outline, dark nuclei and deeply stained cytoplasm (arrows). Notice, gliosis indicated by presence of many glial cells compared with control group (arrow heads) (H\&E X400).
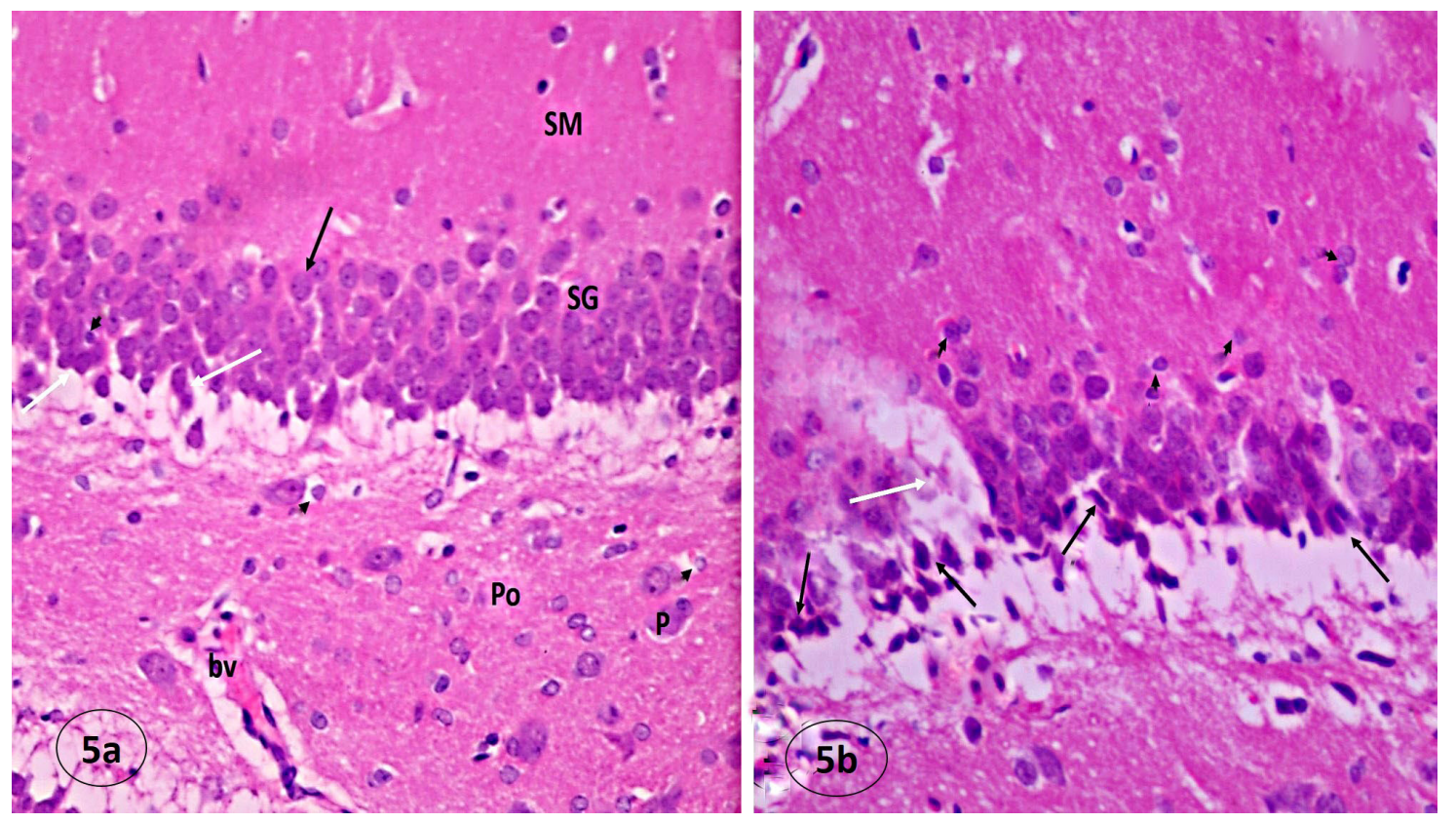

Fig. 5: Photomicrographs of DG subregion (5a) DG of the control group showing its strata; moleculare (SM), granulosum (SG) and polymorphic layer (Po). Granule cells form very closely packed strata. They are small and rounded with pale rounded nuclei (black arrow). Some granule cells appear oval, densely stained (white arrows) and are found in the subgranule zone between SG and Po layers. Notice, large pyramidal neurons (P) in Po layer, glial cells (arrow heads), blood vessels (bv). (5b) DG of iron treated group showing many granule cells become degenerated, irregular in shape, and with deeply stained cytoplasm and nuclei (black arrows). Notice, gliosis (arrow heads) and focal neuronal loss (white arrow) (H \& E x400). 

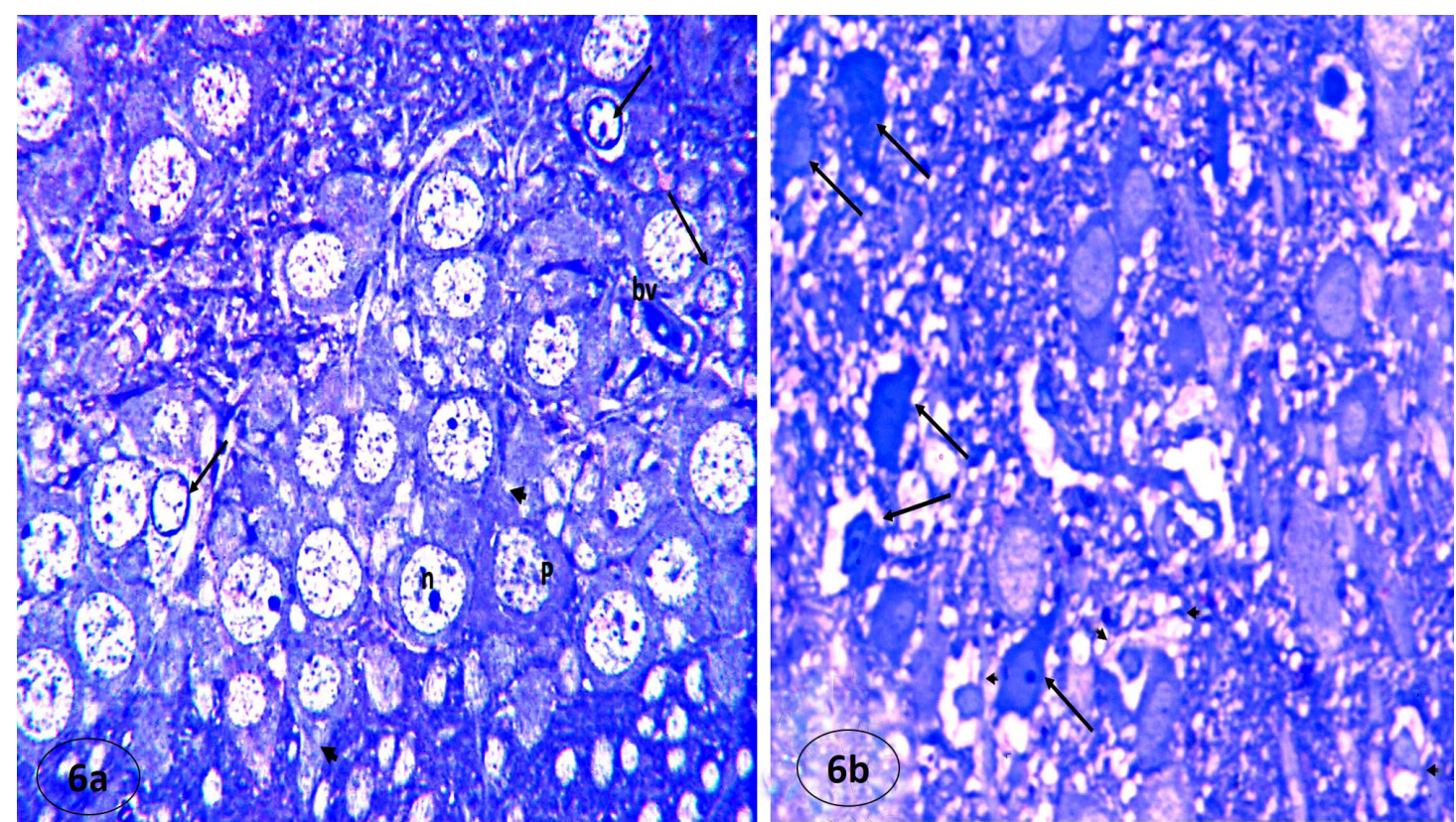

Fig. 6: Photomicrograph of semithin sectionsin CA1 subregion (6a) CA1 of the controlhippocampus showing triangular pyramidal cell bodies (P) with their projecting processes (arrow heads). They have rounded large central vesicular nuclei (n) with prominent nucleoli. Astrocytes (arrows) appear as few scattered cells with pale ovoid nuclei, lightly stained cytoplasm and exhibit some cytoplasmic processes and can be seen near blood vessels (bv). (6b) CA1 of iron treated group showing most neurons are degenerated with irregular outlines, dense ill-defined nuclei and deeply stained cytoplasm (arrows). Many reactive astrocytes with pyknotic nuclei and vacuolated cytoplasm are also noticed (arrow heads) (toluidine blue x1000).
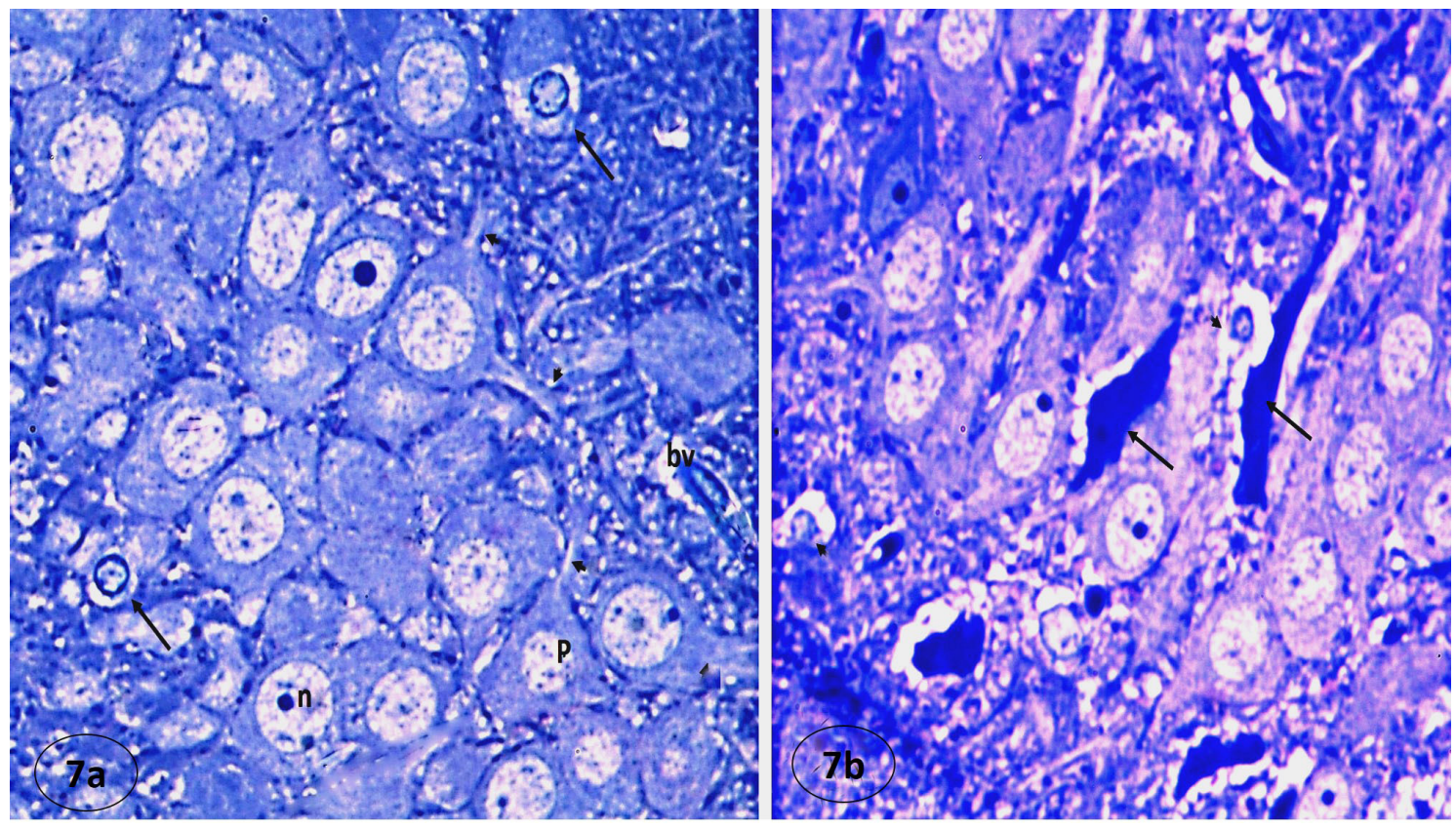

Fig. 7: Photomicrographs of semithin section in CA3 subregion (7a) CA3 of the control group showing large triangular pyramidal cell bodies (P) thatexhibitlarge central vesicular nuclei (n) with prominent nucleoli and some projecting processes (arrow heads). Notice, supporting astrocytes (arrows) and blood vessels (bv).(7b) As noticed in CA1, CA3 of iron treated group showing obvious degeneration of some neurons (arrows) and reactive astrogliosis (arrow heads) (toluidine blue $\mathrm{x} 1000$ ). 

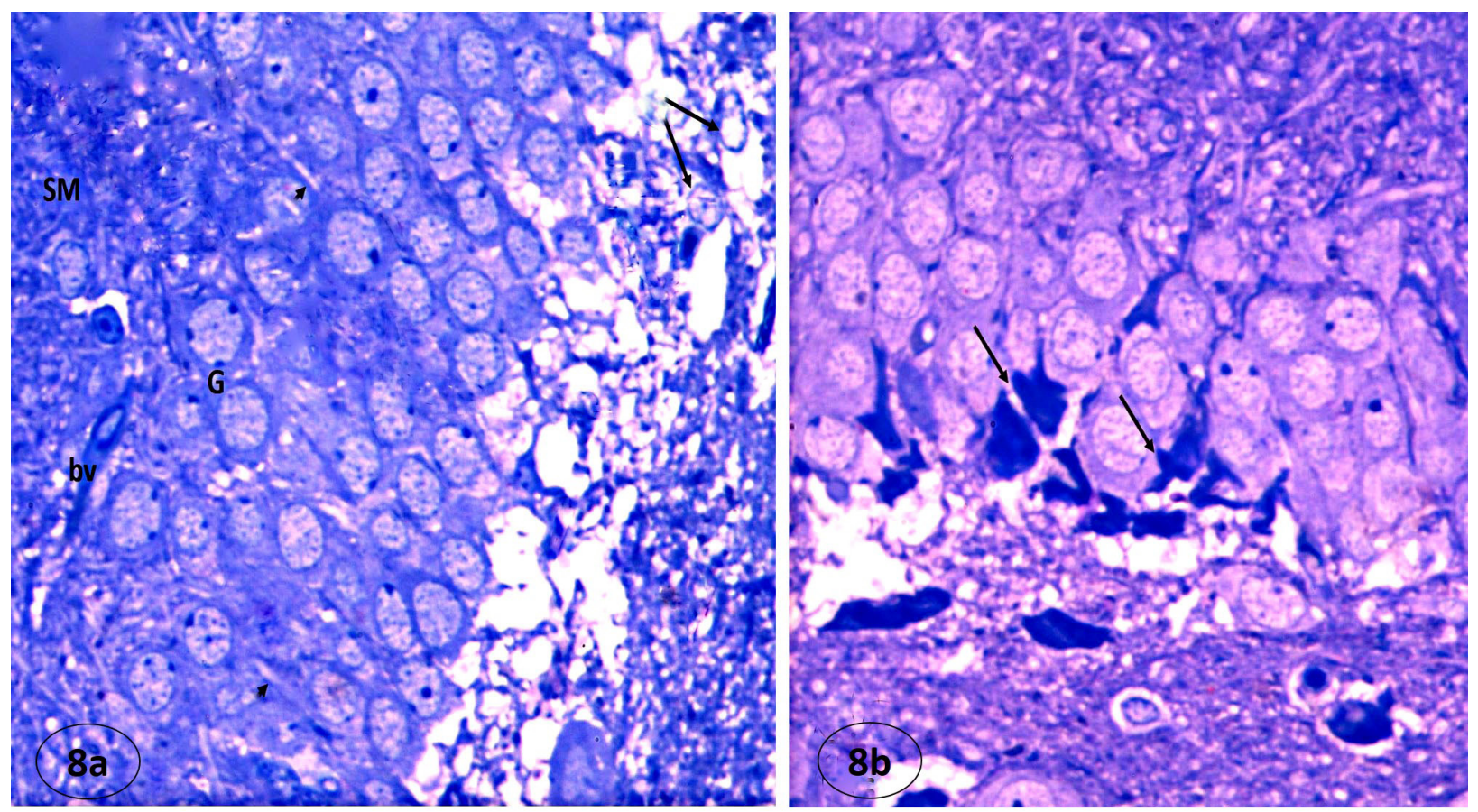

Fig. 8: Photomicrographs of semithin sectionsin DG subregion (8a) DG of the control group showing compactly arranged granule cells (G)that have large pale central vesicular oval or rounded nuclei and scanty cytoplasm. Their apical processes (arrow heads) are projecting towards stratum moleculare (SM). They are supported by astrocytes (arrows) and blood vessels (bv). (8b)DG of iron treated group showing shrinkage and degeneration of many granule cells (arrows) as noticed in other regions of treated hippocampus(toluidine blue x1000).
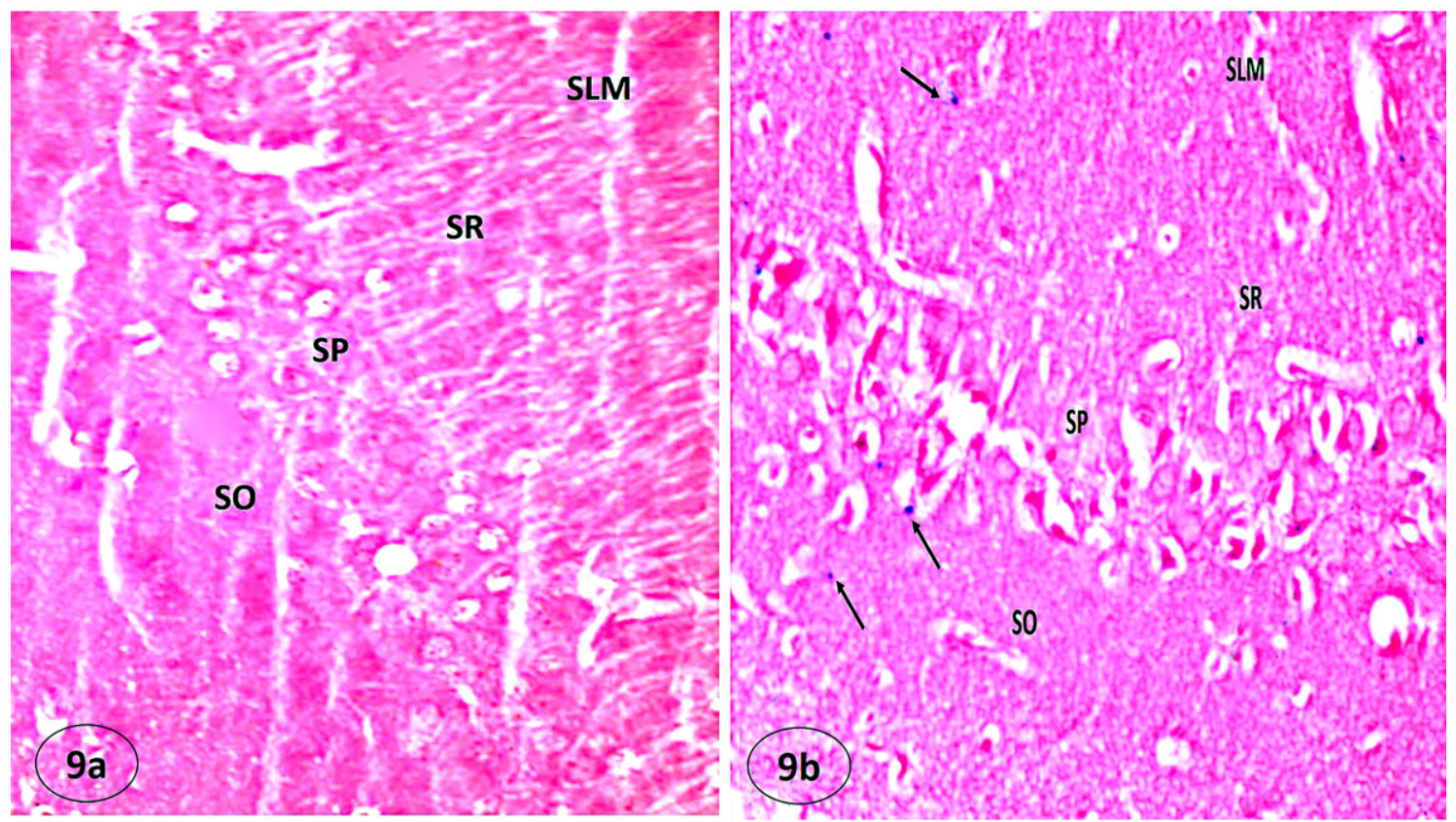

Fig. 9: Photomicrographs of CA1 subregion (9a) CA1 of the control group showing negative reaction for iron particles "no blue precipitate" in all strata (SO, SP, SR and SLM). (9b) CA1 of iron treated group showing positive reaction in the form of blue iron particles precipitate (arrows) seen in all its strata (Prussian blue $\mathrm{x} 400$ ). 

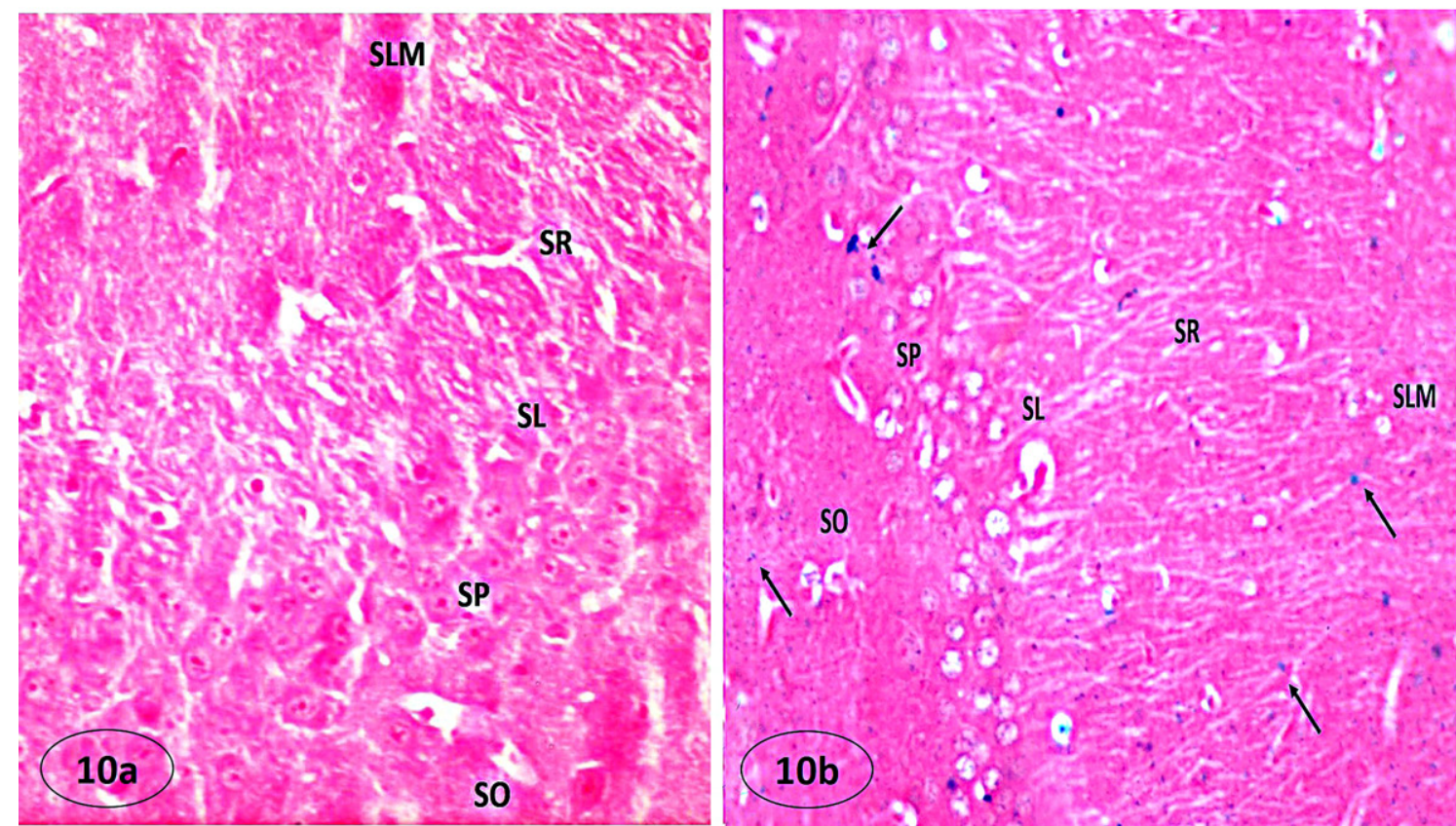

Fig. 10: Photomicrographs of CA3 subregion (10a) CA3 of the control group showing absence of blue iron precipitate in its strata (SO, SP, SL, SR and SLM). (10b) CA3 of iron treated group showing some positive blue iron deposits (arrows) in its different strata (Prussian blue $\mathrm{x} 400$ ).
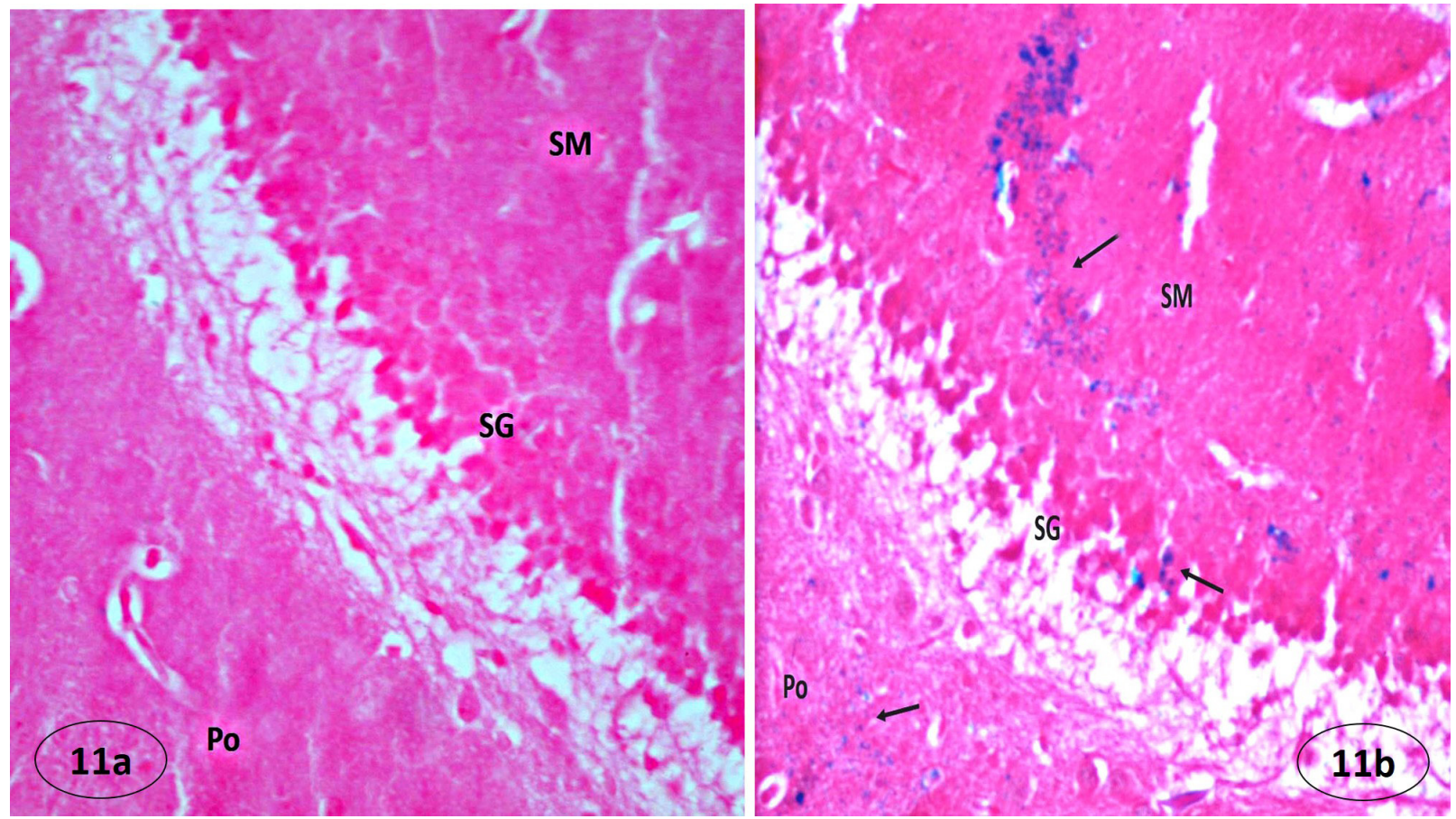

Fig. 11: Photomicrographs of DG subregion (11a) DG of the control group showing nodetectable blue iron precipitate in all strata (SM, SG and PO). (11b) DG of iron treated group showing extensive positive blue iron deposits in all strata (arrows) (Prussian blue x400). 

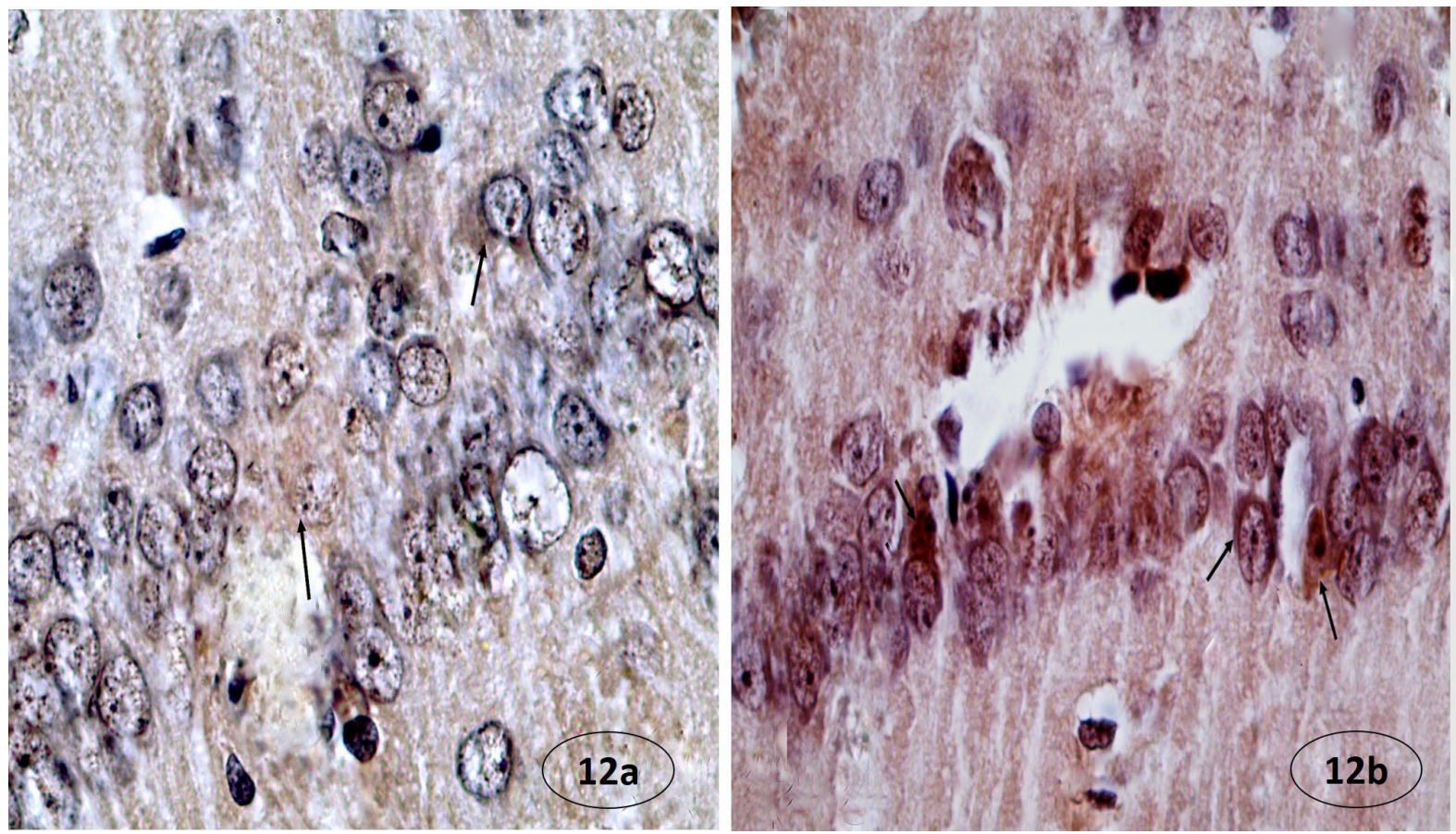

Fig. 12 Photomicrographs of CA1 subregion immunostained with caspase 3 (12a) CA1 of the control group showing almost absent or very weak positive reaction in the cytoplasm of pyramidal neurons (arrows).(12b) CA1 of the iron treated group showing a strong positive reaction in the form of dark brown cytoplasmic granules of manyperikarya (arrows) (Caspase $3 \times 1000$ ).
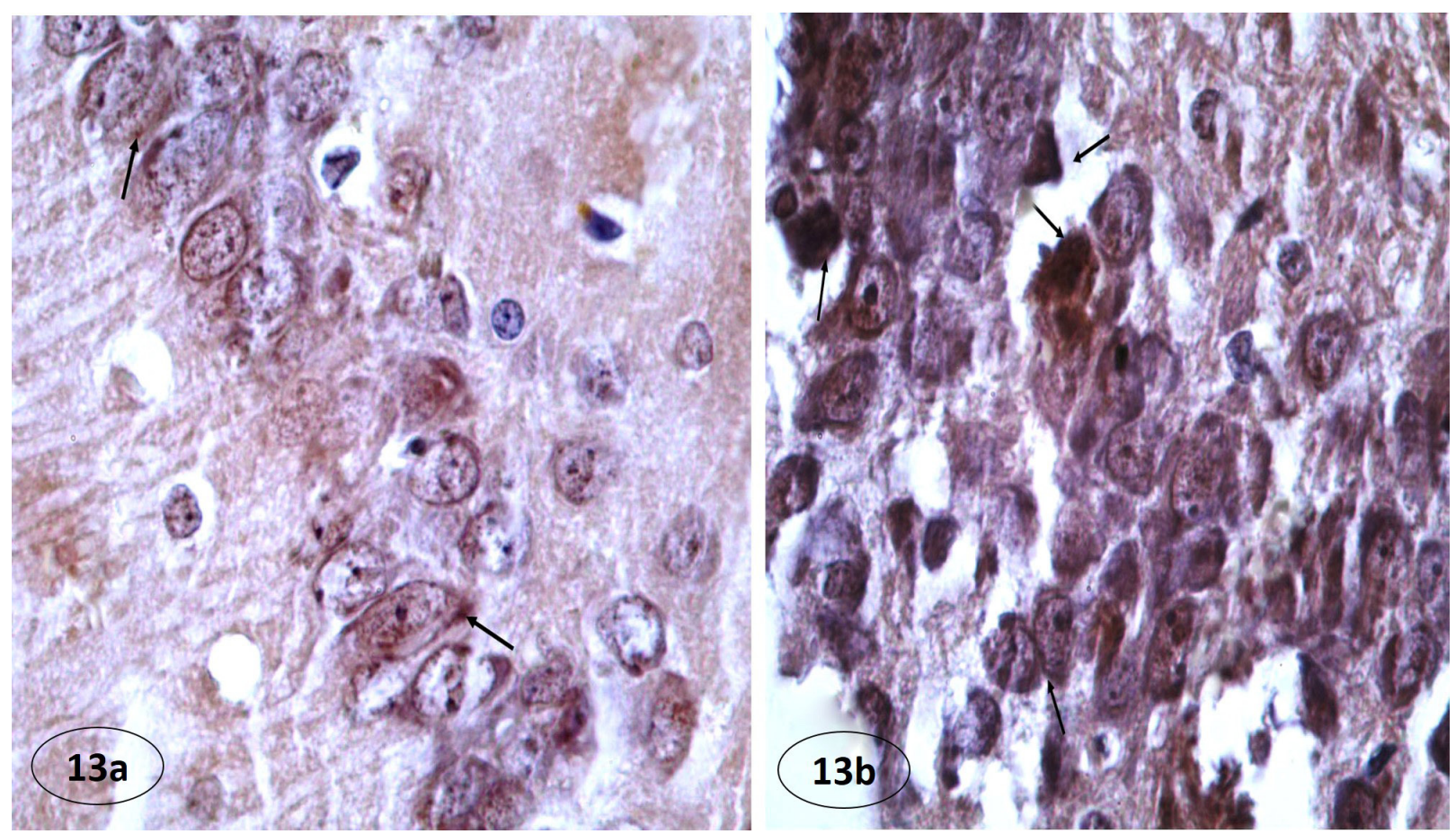

Fig. 13: Photomicrographs of CA3 subregion immunostained with caspase 3 (13a) CA3 of the control group showing its pyramidal neurons exhibit very weak positive cytoplasmic reaction (arrows). (13b) CA3 of the iron treated group showing a strong positive reaction in the cytoplasm of its neurons (arrows) (Caspase 3 x 1000). 

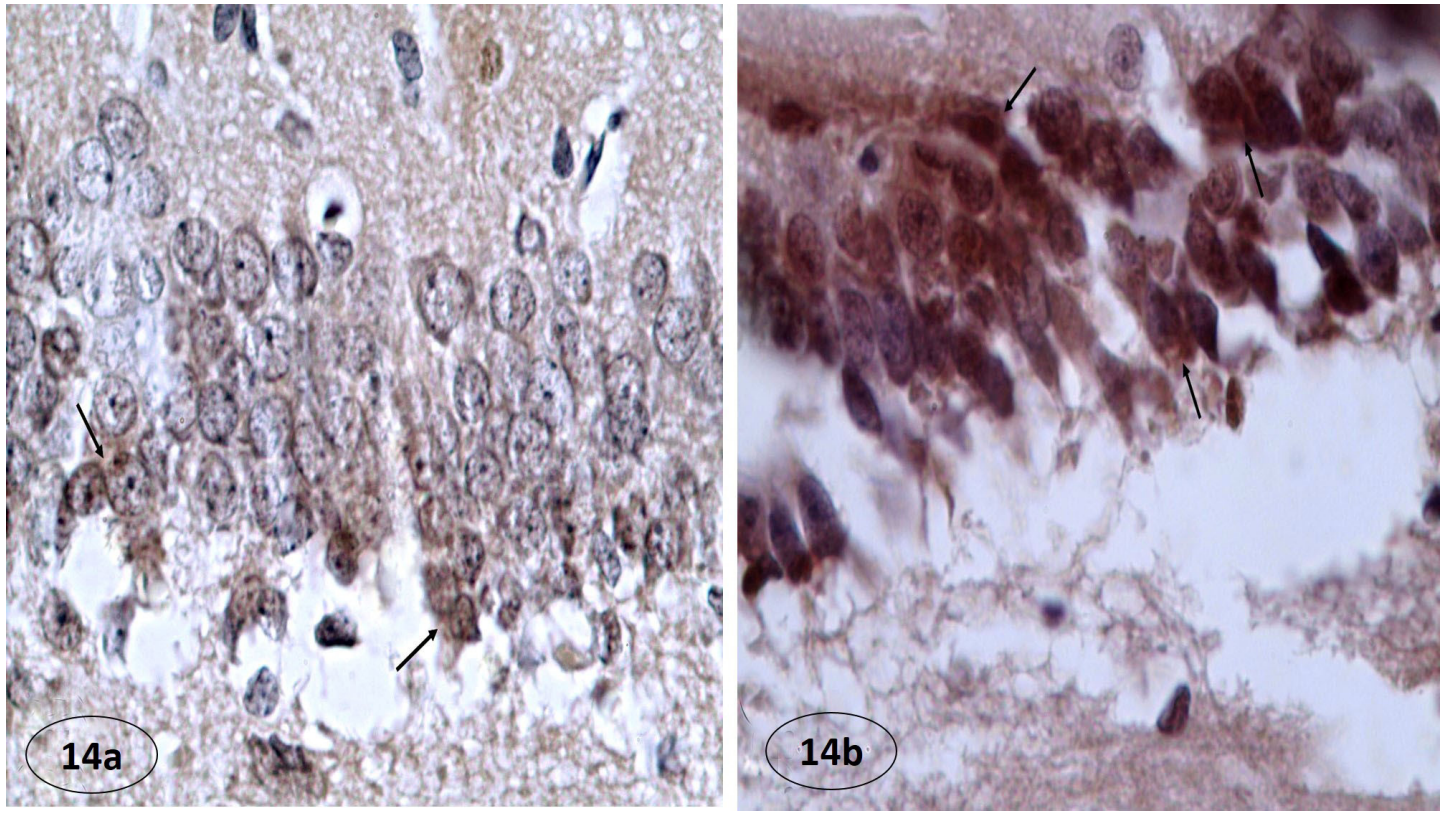

Fig. 14: Photomicrographs of DG subregion immunostained with caspase 3 (14a) DG of the control group showing very weak positive reaction in the cytoplasm of its neurons (arrows). (14b) DG of the iron treated group showing a strong positive dark brownish reaction in the cytoplasm of most pyramidal neurons (arrows) (Caspase 3 x 1000).
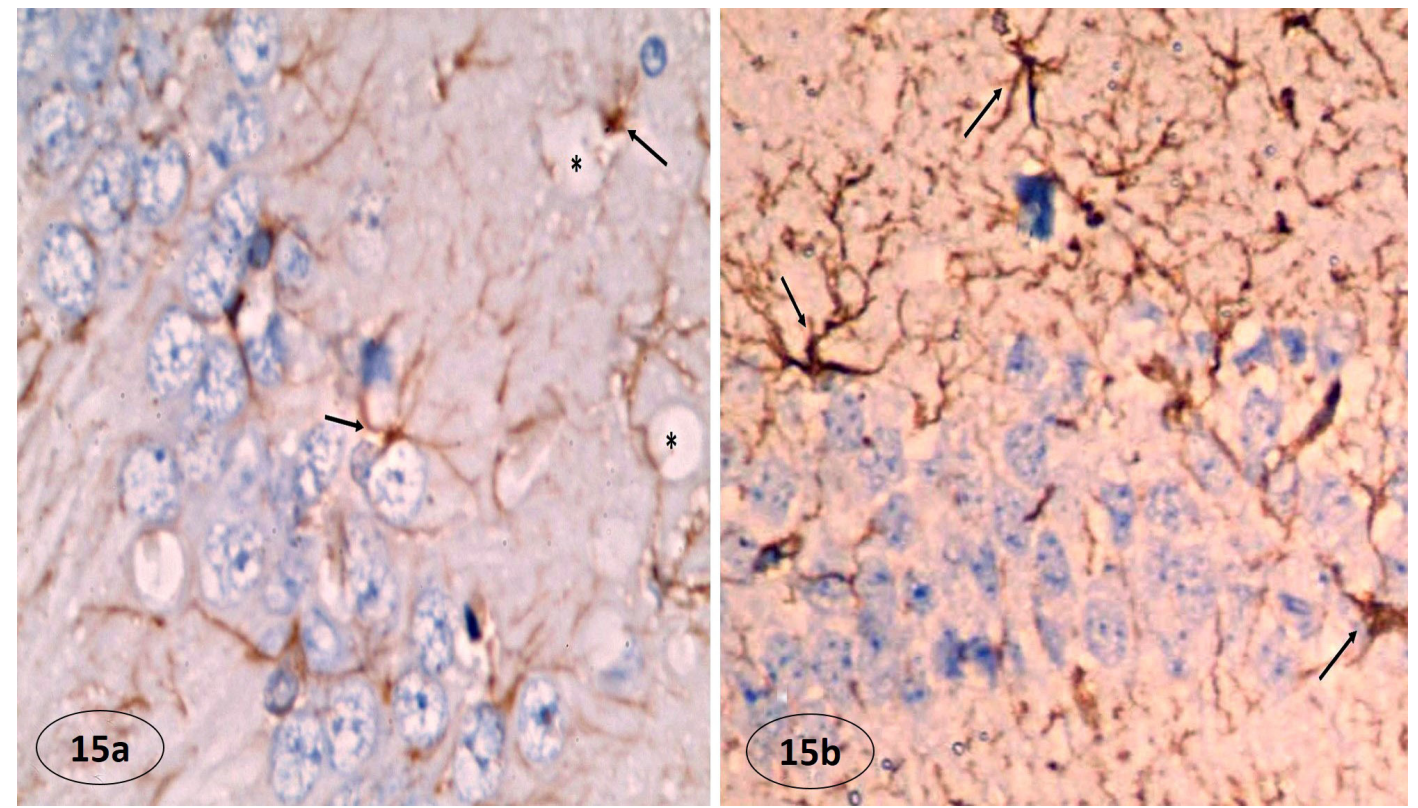

Fig. 15: Photomicrographs of CA1 subregion immunostained with GFAP (15a) CA1 of the control group showing some positively immunostained astrocytes scattered in different layers of CA1. They have small cell body with long thin branching processes (arrows) that extend near the vicinity of blood capillaries $(*) .(15 b)$ CA1 of iron treated group showing more numerous positively stained astrocytesthat exhibit markedly branching processescompared with the control group (arrows) (GFAP x 1000). 


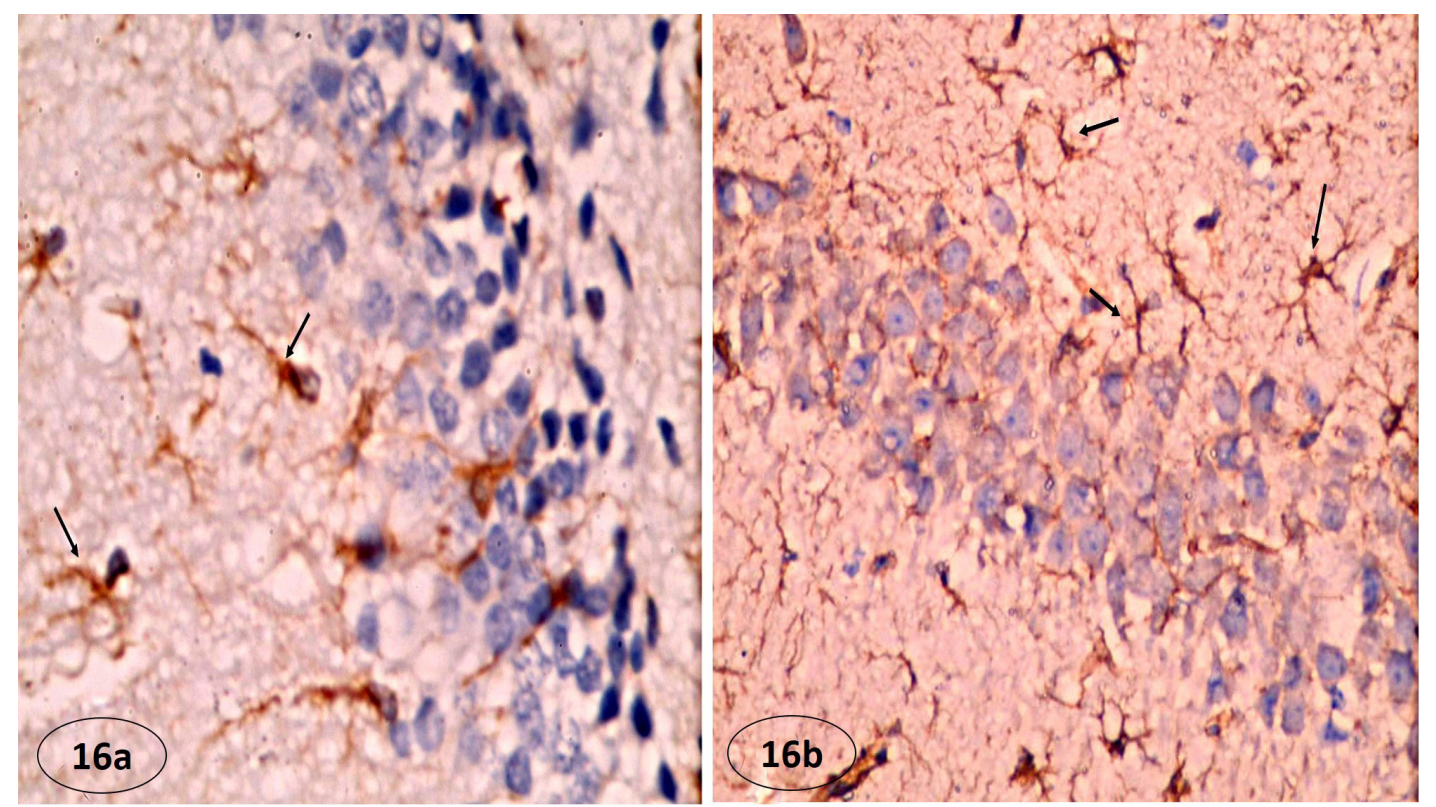

Fig. 16: Photomicrographs of DG subregion immunostained with GFAP (16a) DG of the control group showing few scattered brownish astrocytes with their long processes noticed in different layers of DG (arrows). (16b) DG of iron treated group showing increased frequency of positively stained astrocytes in different layers of DG compared with the control group (arrows) (GFAP x 1000).

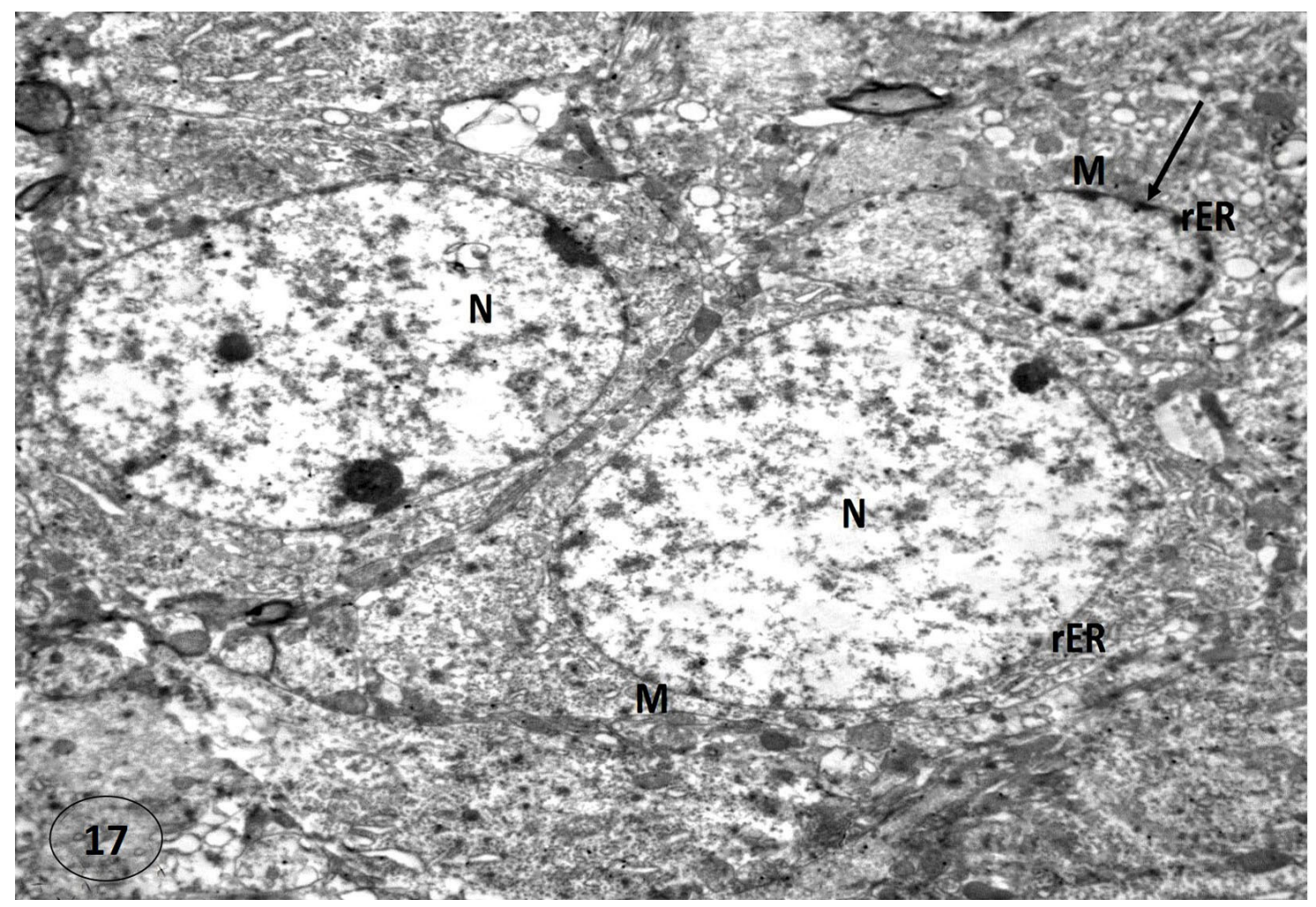

Fig. 17: An electron micrograph of CA1 of the control group showing its pyramidal neurons having large rounded to oval euchromatic nuclei (N) with intact regular nuclear membrane. Its cytoplasmcontains many mitochondria (M) and rER.Astrocyte is noticed adjacent to pyramidal neurons. It has smaller oval euchromatic nucleus with thin rim of peripheral heterochromatin (arrow) and scanty cytoplasm contains few mitochondria (M) and rER (X2900). 


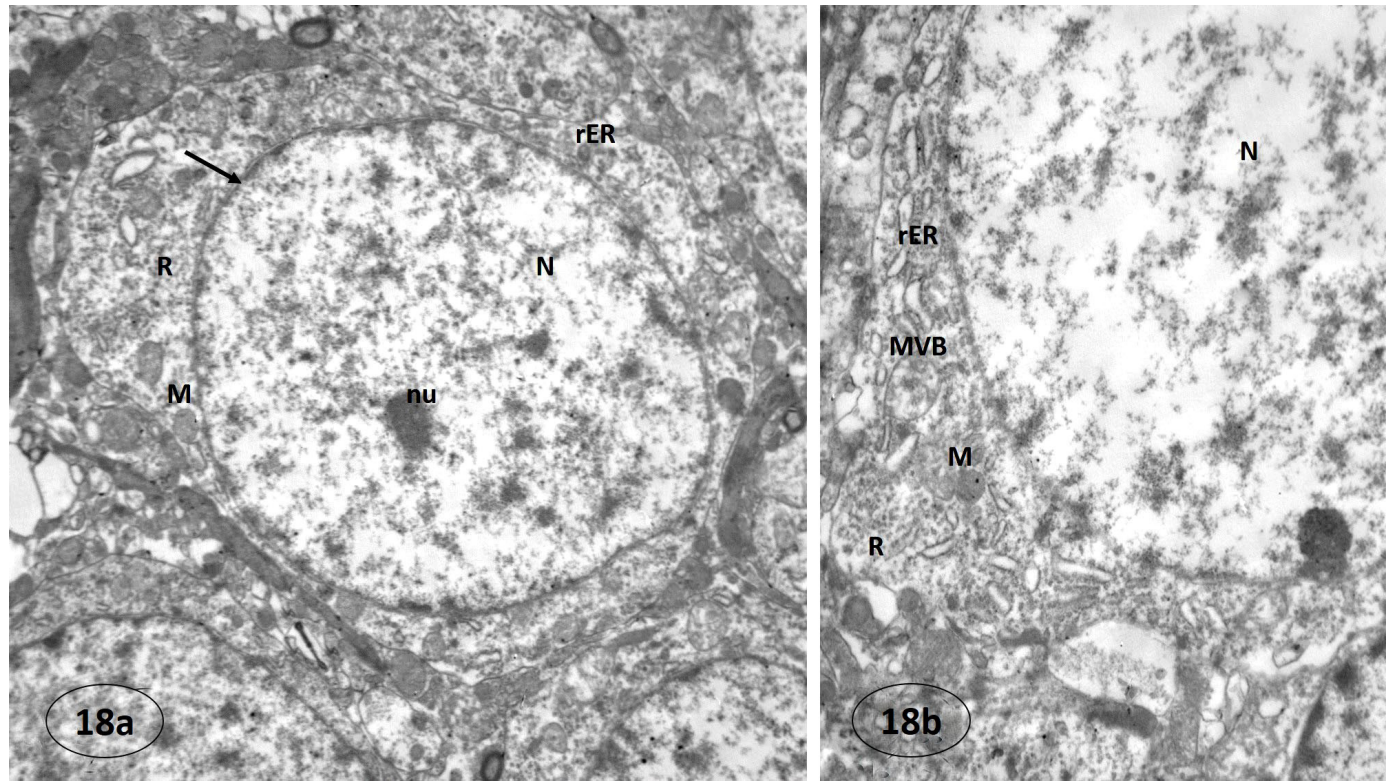

Fig. 18: An electron micrograph of CA1 pyramidal neuron of the control group showing its cytoplasm is rich in free ribosomes (R), mitochondria (M) and $\mathrm{rER}$. Its nucleus $(\mathrm{N})$ is large rounded and euchromatic with prominent nucleolus $(\mathrm{nu})$ and has intact smooth nuclear envelope (arrow) (18a X5800). In 18b; a magnified part of voluminous cytoplasm of CA1 pyramidal neuron containing rER cisternae, free ribosomes (R) and mitochondria (M) with intact cristae. Notice; multivesicular body (MVB) and part of its euchromatic nucleus (N) (X7200).

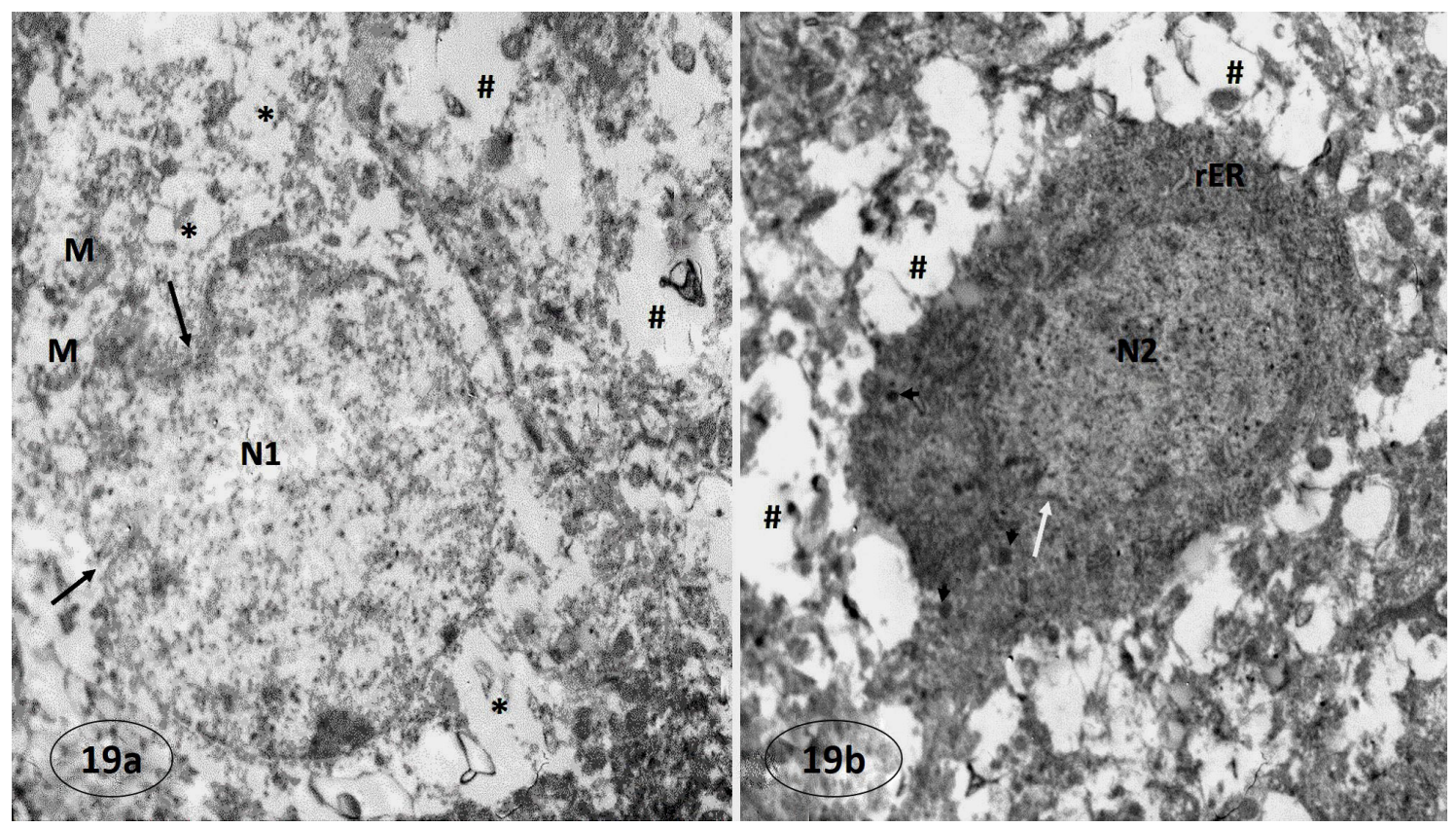

Fig. 19: An electron micrograph of CA1 of iron treated group showing degenerated pyramidal neurons with variable electron density surrounded by wide empty spaces of degenerated neuropil (\#). In 19a, paler degenerating neuron exhibits rarified cytoplasm with complete lysis of most organelles leaving empty spaces $\left(^{*}\right)$. Few remaining mitochondria are seen with partially damaged cristae (M). Nucleus (N1) appears euchromatic with deeply indented outline (black arrows). In 19b; dark degenerated neuron appears small and shrunken with irregular outline. It has electron dense cytoplasm with poorly defined organelles. Some rER cisternae and lysosomes (arrow heads) can be hardly seen in the electron dense cytoplasm. Its nucleus (N2) is shrunken with irregular outline (white arrow) (X5800). 


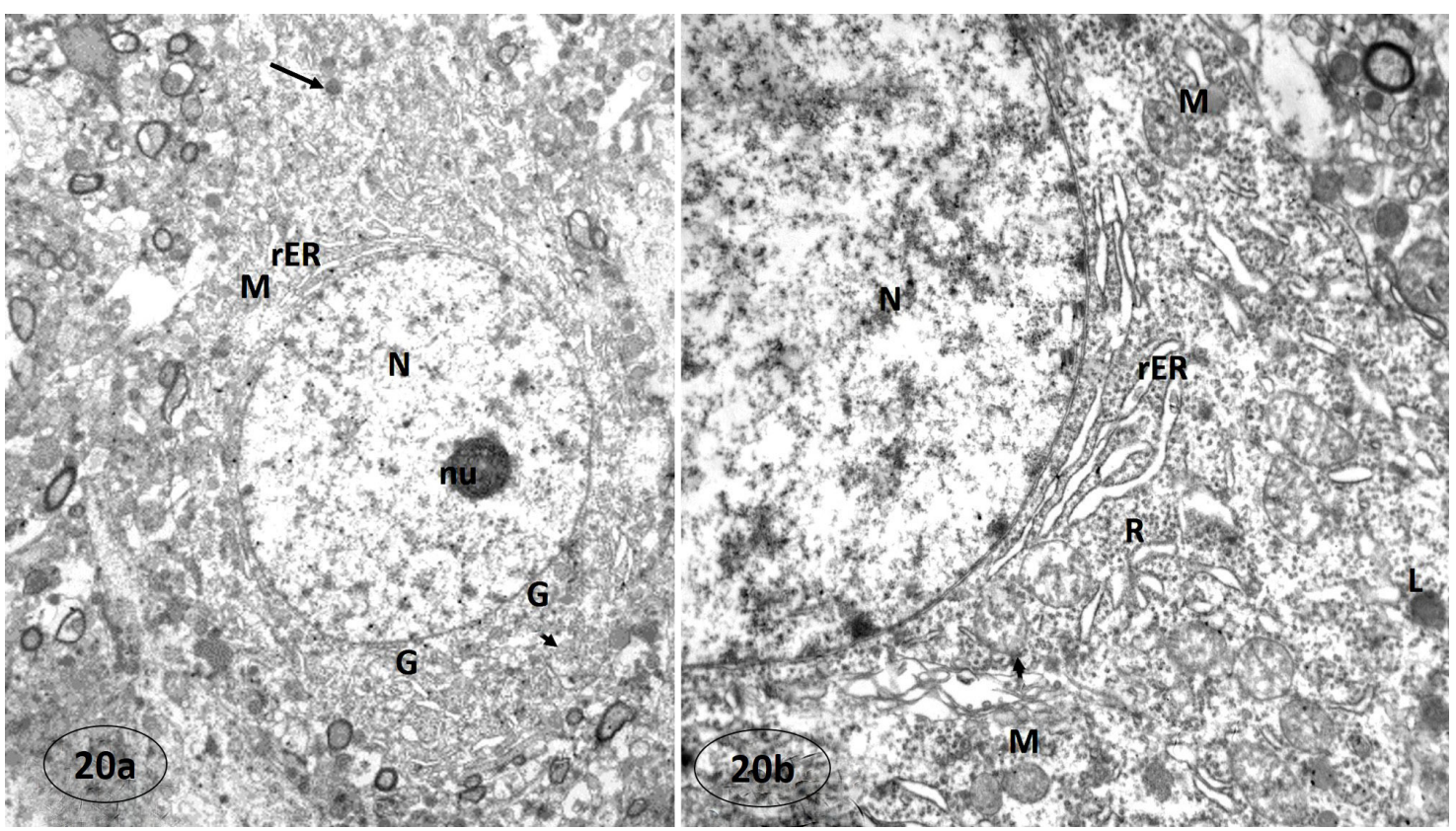

Fig. 20: An electron micrograph of $\mathrm{CA} 3$ of control group showing its pyramidal neuron have large rounded euchromatic nucleus $(\mathrm{N})$ with prominent nucleolus (nu). Its cytoplasm is studded with abundant rER and ribosomes, many mitochondria (M), and well-developed Golgi apparatus (G). Lysosomes (arrow) and multivesicular body (arrow head) are also noticed (20a X2900).In 20b; a magnified part showing its large nucleus with fine dispersed chromatin. Organelles rich cytoplasm contains many rER, free ribosomes (R), mitochondria (M) and multivesicular bodies (arrow head) and lysosomes (L) (X7200).
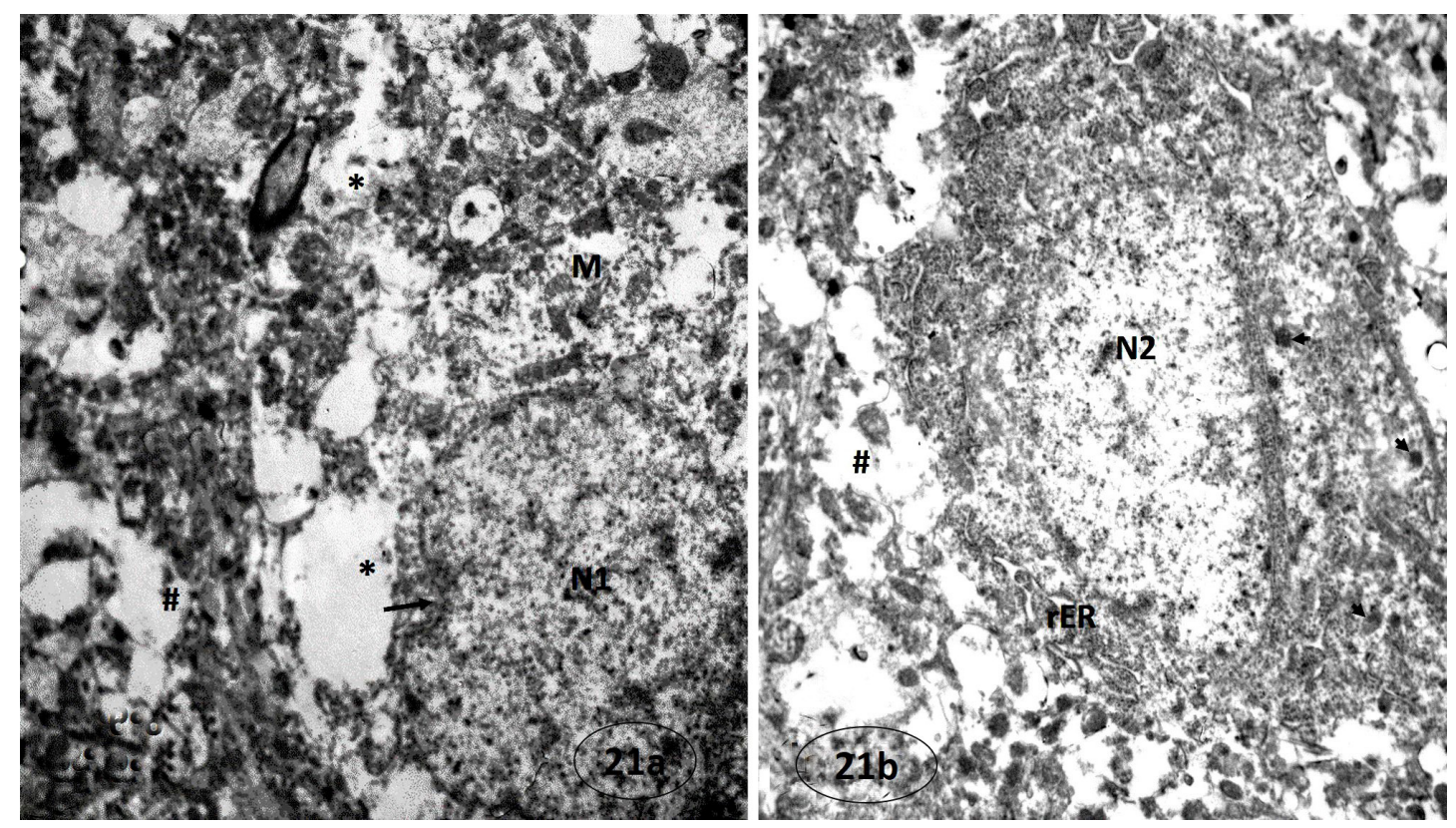

Fig. 21: An electron micrograph of CA3 of iron treated group showing degenerated pyramidal neurons with variable electron density surrounded by wide empty spaces of degenerated neuropil (\#) similar to those seen in CA1 subfield. In 21a; paler degenerating neuron has poorly defined cell membrane and its pale nucleus (N1) appears with deeply indented outline (black arrow). Its rarified cytoplasm has lost most organelles leaving empty spaces $(*)$. Some mitochondria are seen with partially damaged cristae (M). In $21 \mathrm{~b}$; dark smaller irregular degenerated neuron is seen having electron dense cytoplasm with ill-defined organelles except some rER cisternae and many lysosomes (arrow heads). Its nucleus (N2) is small and shrunken (X5800). 


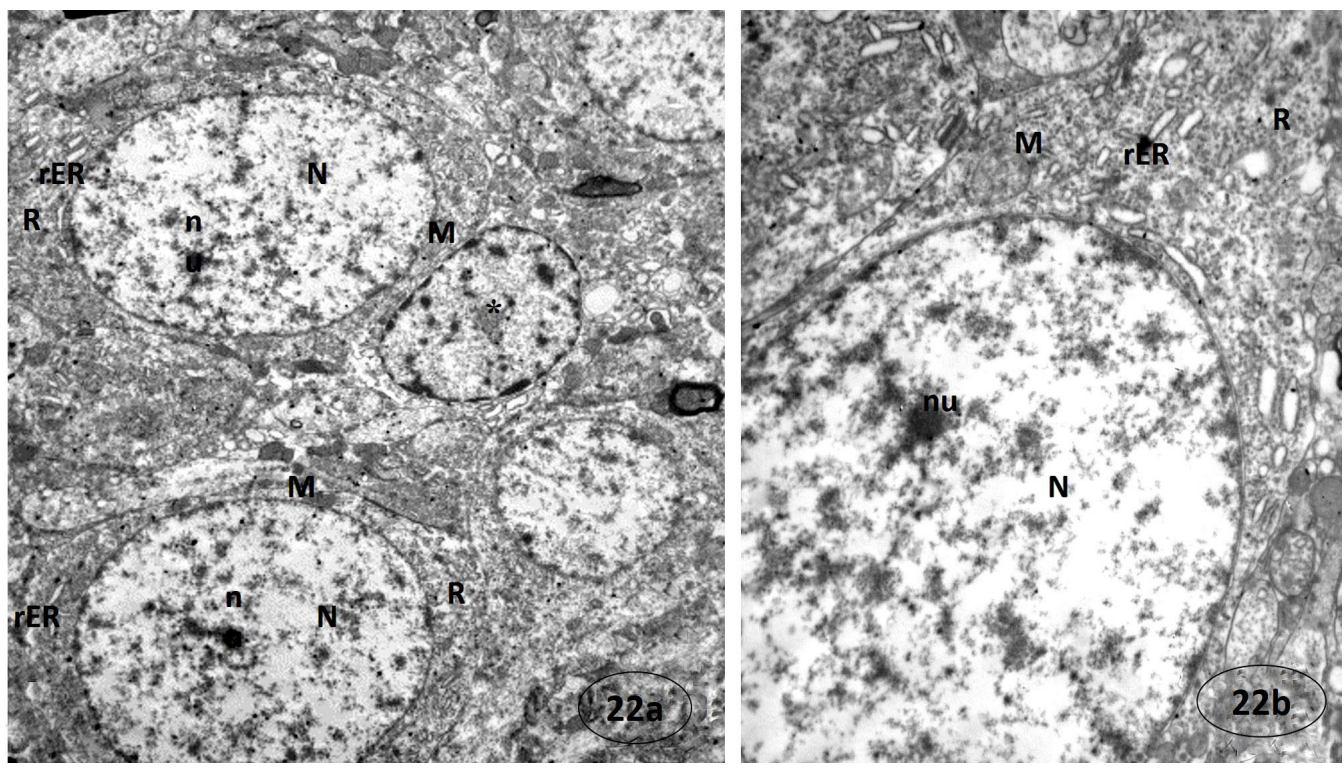

Fig. 22: An electron micrograph of DG of control group showing its granule cells haveoval or rounded nuclei (N) that contain fine dispersed chromatin and prominent small nucleoli $(\mathrm{nu})$. The cytoplasm contains some rER cisternae, free ribosomes (R) and mitochondria (M). Astrocyte $(*)$ is noticed adjacent to neurons having typical ovoid euchromatic nucleus delineated by thin rim of heterochromatin and has scanty cytoplasm (22a X2900). In 22b; a magnified part of granule cell cytoplasm containing rER, free ribosomes and mitochondria and part of its large euchromatic nucleus (N) with its small nucleolus (nu) (X7200).

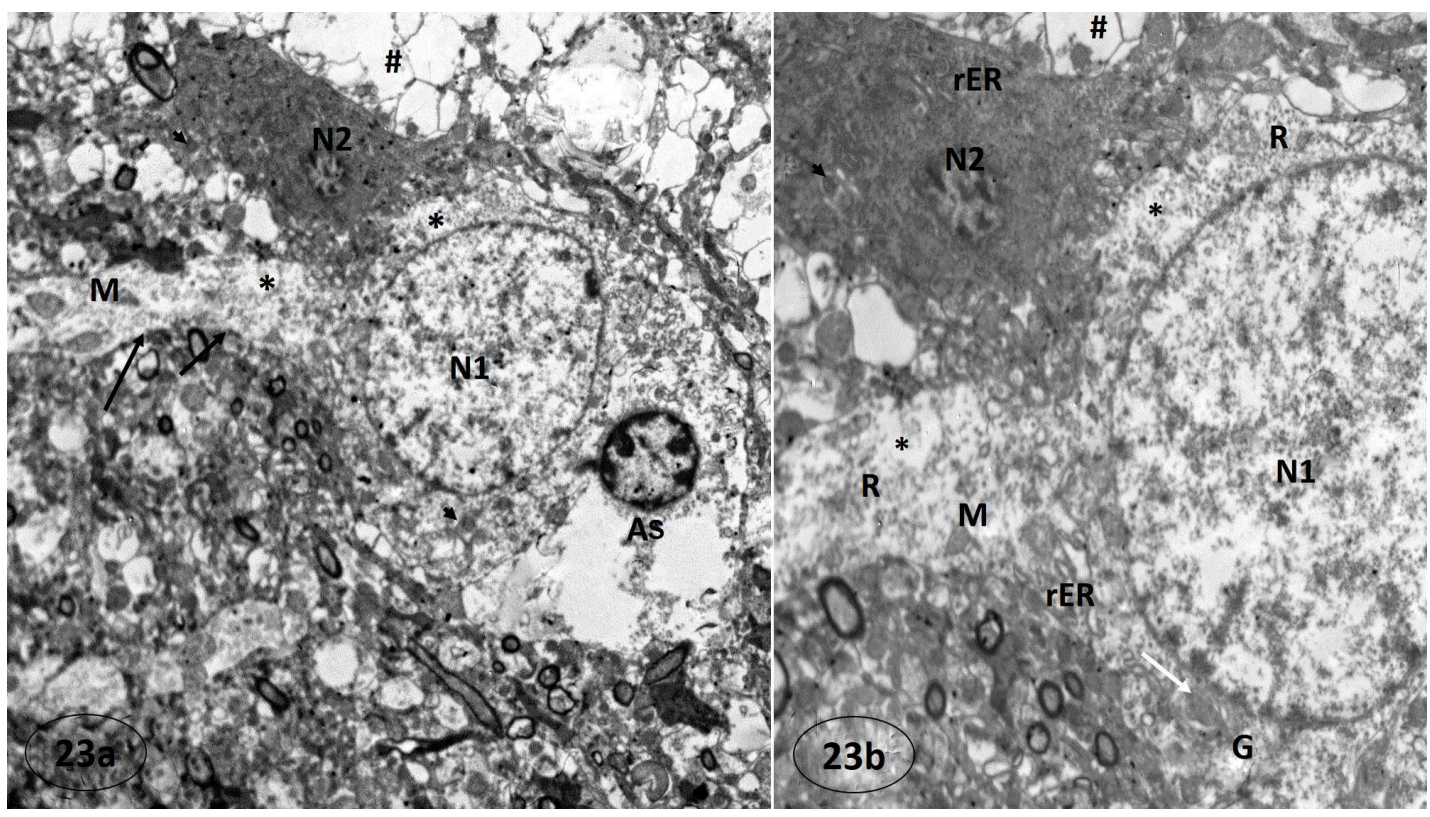

Fig. 23: An electron micrograph of DG of iron treated group showing obvious degeneration of granule cells. Like other studied subregions of treated hippocampus, the degenerating neurons has variable density and surrounded by empty spaces (\#). Paler degenerating neuron appears with its projecting dendrite (black arrows). Its cytoplasm had lost some organelles leaving empty spaces $(*)$. Still some organelles are noticed as deformed mitochondria (M) and some lysosomes (arrow head), Golgi apparatus (G) associated with vesicular structure (white arrow), free ribosomes (R) and some dilated rER cisternae. Its nucleus (N1) appears euchromatic with regular outline. Dark degenerated neuron appears very small and shrunken with deeply dense ill-defined cytoplasm except for some rER cisternae and lysosomes (arrow head). Its nucleus (N2) is markedly shrunken and apoptotic. In (Fig. 23a); reactive astrocyte (As) is seen adjacent to the affected neurons with markedly swollen electron lucent cytoplasm (23a X2900 and 23bX5800). 

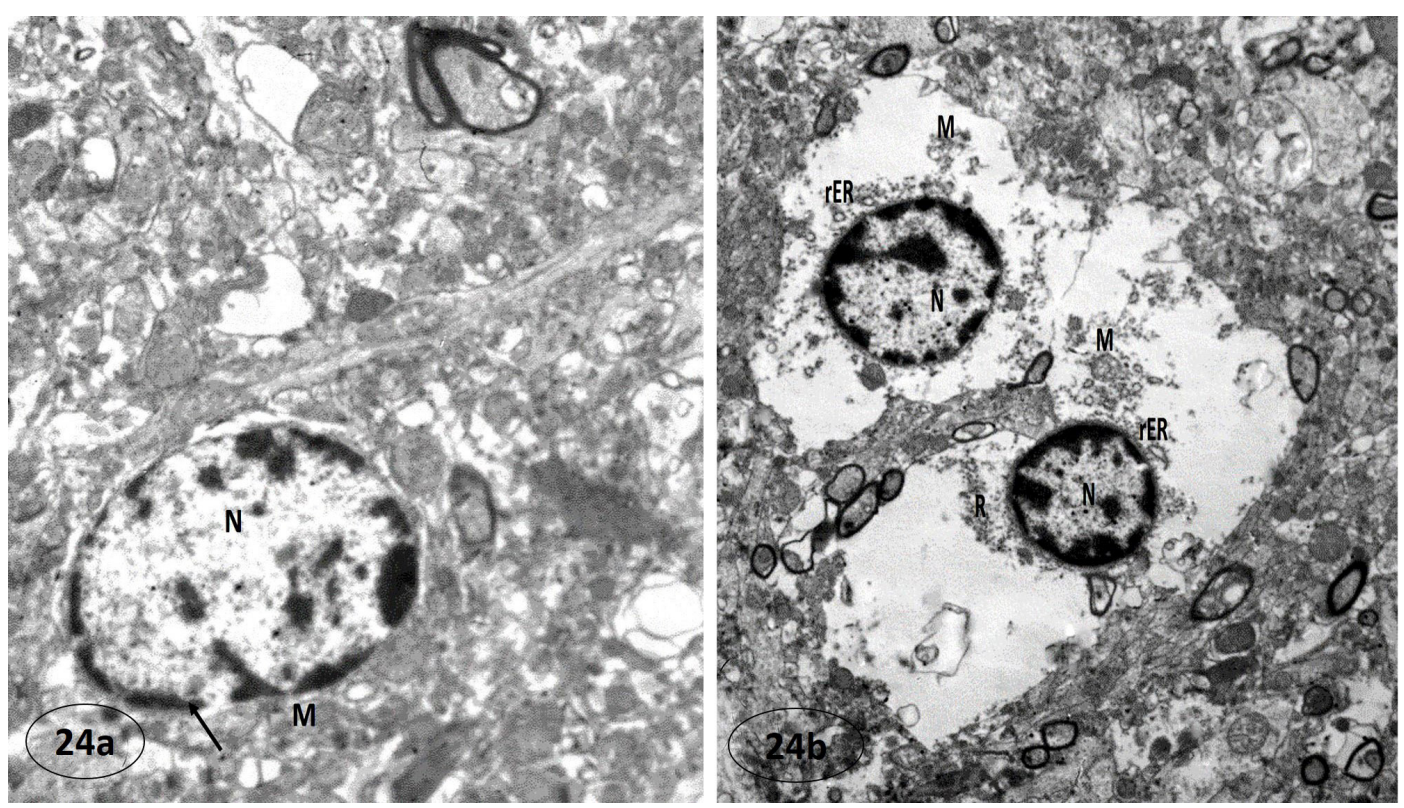

Fig. 24: Electron micrographs of astrocytes (24a) Astrocyte of control group showing its characteristic oval euchromatic nucleus (N) delineated by thin rim of heterochromatin (arrow). Its scanty electron lucent or pale cytoplasm contains few organelles as mitochondria (M) (X5800). (24b) Reactive astrocytes of iron treated group displaying markedly enlarged or swollen electron lucent cytoplasm. They contain few damaged mitochondria (M), few dilated rER cisternae, and free ribosomes $(\mathrm{R})$. Their nuclei $(\mathrm{N})$ appear small with large peripheral and scattered heterochromatin clumps (X5800).
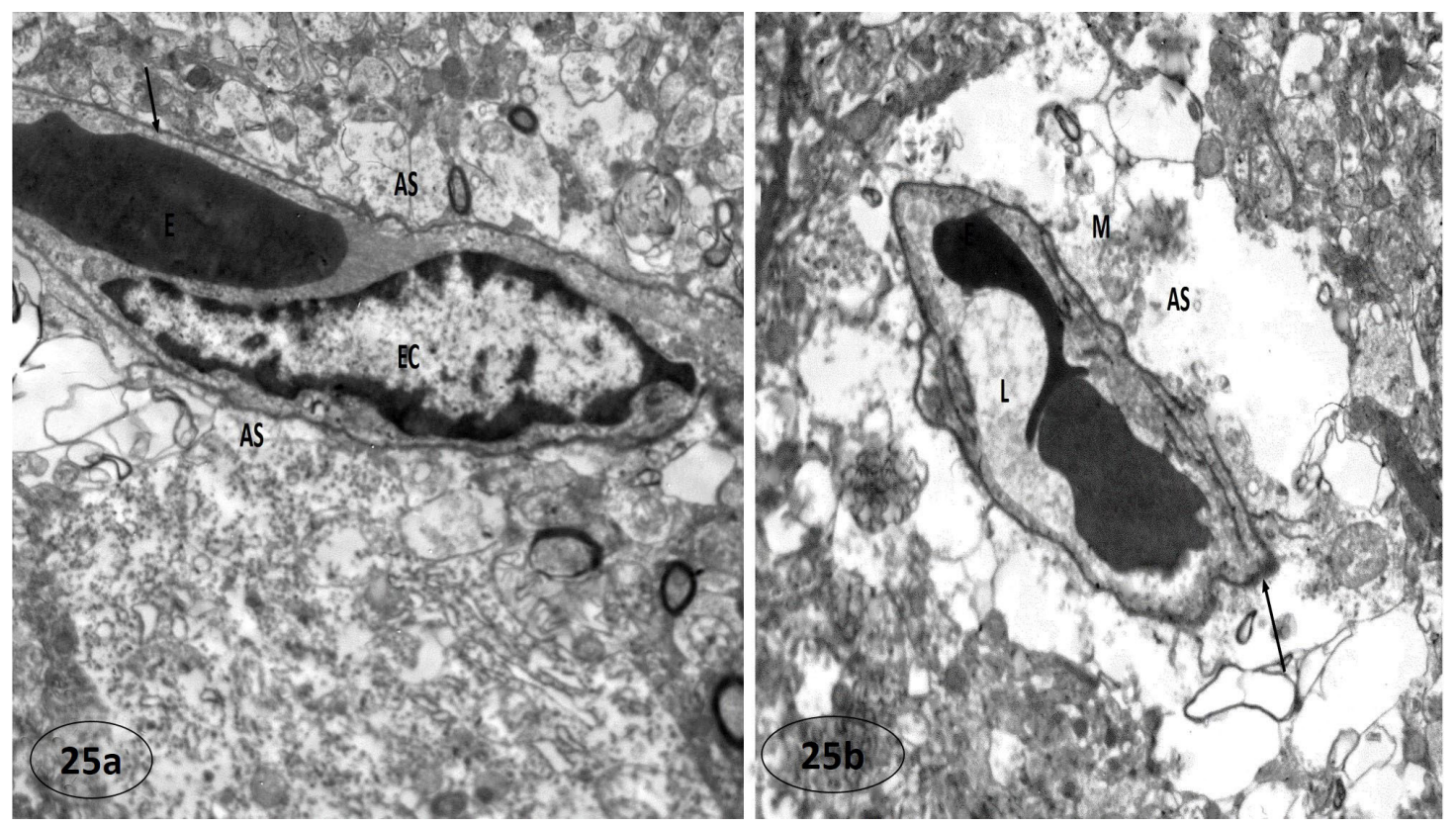

Fig. 25: Electron micrographs of blood capillary (25a) Blood capillary of control group showing its endothelial cell (EC) has flattened nucleus containing heterochromatin clumps. It exhibits intact thin regular basal lamina (arrow) surrounded by astrocyte end feet or processes (AS) and its lumen contains an erythrocyte (E) (X5800). (25b) Blood capillary of iron treated group showing its irregular thickened basal lamina (arrow) and irregular lumen (L) containing deformed erythrocytes (E). It is surrounded by markedly swollen electron lucent astrocytes processes or end feet (AS) containing swollen damaged mitochondria (M) (X5800). 


\section{DISCUSSION}

Iron accumulation was considered a hallmark of many neurodegenerative diseases as Parkinson's and Alzheimer's diseases. Moreover, the association between excess iron concentration in brain and obvious cognitive deficits with aging was also documented ${ }^{[20]}$. In infants and young children, excess iron administration has been reported to disturb linear growth, head circumference and weight gain. Recent concerns are interested in investigating whether early life exposure to excess iron has long run impact on adult brain and its functions ${ }^{[9]}$. In the current study, we especially focused on studying the possible toxic impact of iron overload on developing or growing hippocampus being a critical neural region that is responsible for higher brain functions as cognition, memory, learning and social behavior. Moreover, hippocampus was documented to be highly sensitive to changes in homeostasis and its pyramidal neurons in CA1, CA3 and granule cells in DG are highly vulnerable to oxidative stress damage ${ }^{[21]}$. Indeed, there are some supporting evidences that iron overload disturb cognitive development and memory in both rodents ${ }^{[11]}$ and children ${ }^{[12]}$. One recent follow up study have reported compromised neurodevelopment measures, spatial memory and visual motor integration of children at the age of 10 who were provided with ironfortified formula during infancy ${ }^{[10]}$. However, such studies are still limited and need more investigations. In fact, morphological analysis of possible cellular, subcellular or ultrastructural alternations of hippocampus secondary to excess neonatal iron exposure are not clearly examined yet. As far as we know, it is the first detailed histological and morphometric evaluation of impact of early neonatal exposure to iron overload on adult hippocampal subregions CA1, CA3 and DG using a neonatal rat model. Rodent models are documented to be relevant to study impaired iron metabolism on human ${ }^{[20]}$.

In the present study, iron deposits were detected in all studied subfields of iron treated hippocampus usingPrussian blue stain and were negative in the control hippocampus, indicating that iron over supplementation to neonate rats enhanced its precipitation and accumulation on developing hippocampus. Similar observation was reported in the developing hippocampus of newborn pigs exposed to excess iron and it was associated with disturbance of sociability and social recognition memory of piglets ${ }^{[9]}$. Coinciding with this finding, it is known that fetal and early postnatal period is a critical period that shows rapid brain development and growth in most animals including humans ${ }^{[22]}$. Furthermore, studies on iron metabolism in rat brain showed that iron transport and transferrin binding sites become maximal during postnatal period in parallel with rapid brain growth. Specifically, indeveloping hippocampus, iron import reaches its greatest level (mainly in CA1) immediately before hippocampal growth spurt prior to the postnatal day $15^{[23]}$. At this critical period of development, once iron is absorbed, it become retained in the brain ${ }^{[11]}$. These serious facts could reflect and highlightthe importance of applying strict and proper strategies for iron supplementation and handling to neonates. As this critical period of rapid brain developmentaffects the retained store of brain iron permanently with subsequent possible irreversible hazardous effects later in life. Still, the underlying pathways behind selective accumulation of iron on certain brain regions and not on other regions or organs are unclear and need further investigation.

In the present study, excess iron exposure led to obvious degenerative changes in the principal neurons of iron treated hippocampal subfields CA1, CA3 and DG that was apparently more obvious in CA1 and DG than CA3. Statistical analysis of all studies subfields CA1, CA3 and DG of iron treated group showed significant increase in the number of degenerated dark cells compared with significant decrease in the number of light normal principal neurons. These morphometric data confirmed the obvious degeneration noticed in $\mathrm{H} \& \mathrm{E}$ and semithin sections of all studied subfields of treated group. The affected neurons appeared shrunken with irregular outline and deeply stained cytoplasm and dense ill-defined nuclei. At ultrastructural level, neuronal degeneration was observed that confirm also our light microscope and morphometric observations. Degenerated pyramidal neurons of CA1, CA3 and DG exhibited similar subcellular changes. They were of variable electron density and were surrounded by large empty spaces indicating cell retraction and lysis of surrounding neuropil. Paler degenerated cells had rarified cytoplasm and lost most organelles except for some partially damaged mitochondria and its nucleus was euchromatic and had indented outline. Dark degenerated neurons had small shrunken apoptotic nuclei, hardly defined organelles except for numerous lysosomes and some rER cisternae. Collectively, our light, electron microscope and morphometric data confirmed neurodegeneration and neuronal loss of adult hippocampal principal neurons in CA1, CA3 and DG following neonatal exposure to iron overload. Consistent with that, iron overload has been reported to be a key mediator of neuronal loss in neurodegenerative diseases ${ }^{[24]}$. Indeed, the obvious neuronal loss and degeneration reported in the current study in different hippocampal subfields could be attributed to iron overload induced oxidative stress as previously described in prior studies ${ }^{[25]}$. Meanwhile, another study concluded that iron deposition in rat hippocampus didn't increase oxidative parameter ${ }^{[24]}$. In fact, the obvious neurodegeneration of hippocampal neurons noticed in the current work is against that conclusion and supports the high vulnerability of hippocampus to iron overload induced oxidative damage. Especially, it has been widely proved that excess iron concentration in brain generate free radicals through its participation in Fenton reaction and Haber-Weiss cycle. The resulting ROS react with cellular lipids, DNA and protein causing irreversible neuronal damage ${ }^{[26]}$.

At ultrastructural level, a remarkable finding in the current study was the noticeable mitochondrial loss and 
damage which was in agreement with previous reports ${ }^{[27]}$. Interestingly, this important finding could contribute to more understanding of other possible subcellular mechanisms behind iron induced neurotoxicity in hippocampus. In fact, we hypothesize that excess iron could have direct toxic insult on mitochondria and the resulting disruption of mitochondrial structure and its dynamics may contribute to more neuronal injury and aggravating neurodegeneration. Consistent with that, mitochondria are known essential organelles in all cells and are particularly important in the brain as neuronal survival is highly dependent on mitochondrial derived energy and any mitochondrial deficits lead to neuronal death ${ }^{[25]}$. Moreover, mitochondria have been reported to participate in cellular apoptosis triggered by various stimuli through activation of caspase pathway cascade ${ }^{[28]}$. Accordingly, the currently observed disruption in mitochondrial structure (and subsequently its function) could greatly aggravate hippocampal neuronal cell death. Indeed, iron overload could contribute to direct mitochondrial injury by several ways. It has been found that mitochondrial function is crucially dependent on normal iron supply. Hence, disturbance in iron homeostasis can lead to mitochondrial DNA damage and loss of respiratory capacity ${ }^{[29]}$. Also, previous efforts explained that iron overload triggers changes in both mitochondrial and nuclear DNA through induction of oxidative stressand it could evoke mitochondrial fragmentation through activation of calcineurin, a factor involved in mitochondrial fission and subsequent mitochondrial fragmentation ${ }^{[25]}$.

Interestingly as evident in our electron microscope images, there was many lysosomes were noticed in the cytoplasm of degenerated neurons of all studied subfields of iron treated hippocampus. In fact, as mentioned earlier iron generated oxidative stress contribute to neuronal loss, interfere with cellular signaling and lead to nuclear and organelle damage ${ }^{[26]}$, however, still the increased number of lysosomes is greatly questionable. A recent study is consistent with our finding and the authors postulated that in case of aging and neurodegeneration, upregulation of lysosomal genes takes place andthe increased number of lysosomes is considered a protective machinery aiming to struggle for neuronal survival against oxidative stress mediated neuronal damage in such $\operatorname{cases}^{[30]}$.

Caspase family members are well known proteolytic enzymes involved in activation, mediation and execution of apoptosis. Among those members, caspase 3 especially play a critical role in activation and mediation of neuronal apoptosis with subsequent neurodegeneration ${ }^{[31]}$. In the present study, iron treated hippocampal CA-DG subfields showed increased immunoreactivity to caspase 3 . This finding confirms similar observation noticed in adult rat hippocampus and cortex following neonatal exposure to excess iron ${ }^{[11]}$. Based on this remarkable finding, we suggest that iron overload could evoke neurodegeneration in hippocampus through caspase 3 mediated activation of neuronal apoptosis (programmed cell death). In agreement with that, previous effort concluded that Fenton reaction and reactive oxygen species which are released as a result of iron induced oxidative stress can mediate signals that stimulate caspase activation and initiation of neuronal apoptosis $^{[11]}$.

Astrocytes are crucial glial cells in the central nervous system (CNS) that ensure survival of neurons through provision of structural and metabolic support and regulation of synaptic microenvironment. They play an important role in maintenance of blood brain barrier, vascular reactivity, energy metabolism and protection from oxidative stress and reactive oxygen species ${ }^{[32]}$. They react to any CNS insults by overexpression of glial fibrillary acid protein (GFAP) aiming to protect precious neurons through uptake of excitotoxic glutamate, production of neuroprotective adenosine and antioxidant glutathione and limiting inflammatory reaction ${ }^{[33]}$. In the current study, reactive astrogliosis was noticed in all studied CA-DG subfields of treated hippocampus and was manifested as increase in astrocyte number or frequency and increased immunoreactivity to GFAPcompared with control group. Similar observation was previously reported ${ }^{[27]}$. Indeed, we suggest that astrocytic gliosis noticed in the current study could be attributed to toxic insult of iron on hippocampal neurons with subsequent neuronal death. In this regard, it has been documented that astrocytes undergo activation and complex morphofunctional remodeling in case of any brain insults ${ }^{[27]}$. They appear highly resistant to toxicity and have the capability to proliferate greatly unlike neurons. Astrocytic reaction aims to provide more protection to viable neurons ${ }^{[27]}$ and also to take over the spaces left after neuronal death by forming glial scar tissue as a reparative process $^{[11]}$. In fact, a prior effort by Pelizzoni and his colleagues $(2013)^{[32]}$ have reported that under normal condition, astrocytes also function in regulation of brain iron uptake and homeostasis through blood brain barrier (BBB) and are responsible for iron redistribution among neurons. They added that even under iron overload mediated stress, astrocytes are able to buffer excess iron and prevent spill in of iron into synaptic cleft, thereby protecting neuronal cells from potentially harmful iron overload. Unfortunately, our observation revealed obvious degeneration of reactive astrocytes at ultrastructural level which was manifested as markedly swollen cytoplasm containing few damaged mitochondria, dilated rER cisternae and free ribosomes. This could indicate that iron overload ultimately could have diet toxic effect on astrocytes (perhaps through oxidative stress), thereby disrupts astrocytic protective function and its iron buffering action and so contribute more and aggravate iron overload induced neuronal damage. Indeed, further studies are highly recommended to investigate the underlying mechanism behind astrocyte damage noticed in the current work.

Blood brain barrier (BBB) is considered a pivotal protective factor that normally maintains optimal ionic homeostasis and prevent leakage of any harmful insult to brain tissue including excess iron ${ }^{[34]}$. In the current study, there was noticeable disruption on the structure of BBB of 
iron treated group. In this sense, it has been reported that in the developing brain, BBB exhibits higher permeability due to its immaturity, thereby higher susceptible to ion influx and leakage to brain parenchyma ${ }^{[35]}$. This fact might help in understanding why developing hippocampus as noticed in the current study has high affinity to accumulate iron deposits on its tissue. Interestingly and consistent with our finding, previous studies have reported that iron overload itself has direct toxic effect on BBB by enhancing loss of tight junction proteins "occludins and zonula occludins-1" and degeneration of vascular endothelial cells ${ }^{[36]}$. Moreover, and based on our ultrastructural results, we can add that the observed astrocyte damage in the current study could be also an important etiological factor in the disruption of $\mathrm{BBB}$. We also hypothesize that disruption of $\mathrm{BBB}$ could participate in more and more aggravation of iron induced neuronal damage due to unavoidable and uncontrollable free leakage and entry of any hazardous stimuli to hippocampal tissue.

In the current study, according to our observation of different studied regions of treated hippocampus, we noticed that CA1 and DG apparently showed more cellular damage than CA3. A finding which might reflect differential vulnerability of hippocampal subregions to iron mediated oxidative damage. Indeed, the susceptibility of different hippocampal subfields CA-DG to oxidative stress damage was previously discussed and it is still debated $^{[21]}$. Some authors proposed that CA1 neurons are more vulnerable than others ${ }^{[37]}$ while others have suggested $\mathrm{CA} 3$ and DG as most prone to oxidative damage ${ }^{[21,38]}$. Although we recommend further investigation to clarify more the potentiality of hippocampal subfields to iron evoked oxidative damage and the possible underlying mechanisms behind that. But, at the end, all subfields have crucial functions and whatever the most affected area, the net result will be serious consequences on the whole hippocampal function.

Collectively, Neonatal iron administration could contribute to late and permanent hippocampal neurodegeneration in adult. Accordingly, Strict precautions and optimal strategy of iron supplementation to neonates and young children have to be applied. It is highly recommended now to consider the assessment of iron level in case of any neuroglial deficit observed in infant and children and chelation therapy could be essential in such cases. Further studies are really recommended to explore and understand the underlying mechanisms behind excess intracellular influx of iron by hippocampus following high neonatal iron exposure which ultimately induce oxidative damage.

\section{CONCLUSION}

Our findings confirmed that neonatal iron over supplementation caused its precipitation and subsequent toxicity and profound neurodegeneration in hippocampal CA1, CA3 and DG subregions that could explain memory and cognitive deficits noticed in previous studies. Here, neurodegeneration could be multifactorial, due to caspase 3 mediated apoptosis, direct toxic insult to astrocyte and $\mathrm{BBB}$ and mitochondrial disruption.

\section{CONFLICT OF INTEREST}

There are no conflict of interest.

\section{REFERENCES}

1. Aggleton JP. Multiple anatomical systems embedded within the primate medial temporal lobe: Implications for hippocampal function. NeurosciBiobehav Rev. 2012; 36(7): 1579-1596.

2. Wang $X$ and Michaelis EK. Selective neuronal vulnerability to oxidative stress in the brain. Front Aging Neurosci (2010); 2:12.

3. Blasco G, Puig J, Daunis-Estadella J, Monila X, Xifra G, Fernandez-Aranda F, Pedraza S, Ricart W, Portero-Otin M and Fernandez-Real JM. Brain iron overload, insulin resistance, and cognitive performance in obese subjects: a preliminary MRI case-control study. Diabetes Care (2014); 37(11): 3076-83.

4. Schenck JF and Zimmerman EA. High-field magnetic resonance imaging of brain iron: birth of a biomarker? NMR Biomed. (2004); 17: 433-445.

5. Zecca L, Youdim MB, Riederer P, Connor JR, Crichton RR. Iron, brain ageing and neurodegenerative disorders. Nat Rev Neurosci (2004); 5(11):863-873.

6. Andersen $\mathrm{HH}$, Johnsen $\mathrm{KB}$ and Moos $\mathrm{T}$. Iron deposits in the chronically inflamed central nervous system and contributes to neurodegeneration. Cell Mol Life Sci (2014); 71: 1607-1622.

7. Lonnerdal B. Excess iron intake as a factor in growth, infections, and development of infants and young children. Am J Clin Nutr (2017); 106:1681S-7S.

8. Wessling-Resnick M. Excess iron: considerations related to development and early growth. Am J Clin Nutr (2017); 106:1600S-5S.

9. Ji P, Lonnerdal $\mathrm{B}$, Kim $\mathrm{K}$ and Jinno $\mathrm{CN}$. Iron over supplementation cases hippocampal iron 
overloading and impairs social novelty recognition in nursing piglets. J Nutr (2019);149: 398-405.

10. Lozoff B, Castillo M, Clark KM, and Smith JB. Iron-fortified vs low iron infant formula: developmental outcome at 10 years. Arch PediatrAdolesc Med (2012);166: 208-15.

11. Miwa CP, de Lima MN, Scalco F, Vedana G, Mattos R, Fernandez LL, Hilbig A, Shroder $\mathrm{N}$ and Vianna MR. Neonatal iron treatment increases apoptotic markers in hippocampal and cortical areas of adult rats. Neurotox Res (2011): 19(4): 527-35.

12. Lozoff B, Castillo M, Clark KM and Smith JB. Follow up of a randomized controlled trial of iron fortified $(12.7 \mathrm{mg} / \mathrm{L}) \mathrm{vs}$. low iron $(2.3 \mathrm{mg} / \mathrm{L})$ infant formula: developmental outcome at 10 years. Arch PediatrAdolesc Med (2012): 166(3): 208-215.

13. Amin SB, Myers G and Wang H. Association between neonatal iron overload and early human brain development in premature infants. Early Hum Dev (2012); 88(8): 583-587.

14. Buonocore G, Perrone $\mathrm{S}$ and Bracci R. Free radicals and brain damage in the newborn. Biol Neonate (2001); 79(3-4):180-186.

15. Maaroufi K, Ammari M, Jeljeli M, Roy V, Sakly $M$ and Abdelmelek $H$ : Impairment of emotional behavior and spatial learning in adult Wistar rats by ferrous sulfate. PhysiolBehav (2009); 96(2): 343-9.

16. Bancroft JD, and Layton C. Theory and practice of histological techniques.7th ed.: Churchill Livingstone of Elsevier, Philadelphia; (2013). 172-214.

17. Faherty CJ, Xanthoudakis S and Smeyne RJ. Caspase 3 dependent neuronal death in the hippocampus following kainic acid treatment. Mol Brain Rec (1999); 70: 159-163.

18. Hayat MA. Chemical fixation. In: Principles and techniques of electron microscopy: biological applications. 4th ed. Edinburg, UK: Cambridge University Press (2000); 485.

19. Doaa M. Abo El-Khair, Fatma El-Nabawia A. El-Safti, Hanaa Z. Nooh, Abeer E. El-Mehi.A comparative study on the effect of high cholesterol diet on the hippocampal CA1 area of adult and aged rats. Anat Cell Biol (2014); 47(2): 117-26.

20. Agrawal S, Berggren KL, Marks E and Fox
JH. Impact of high iron intake on cognition and neurodegeneration in humans and in animal models: a systemic review. Nutr Rev (2017); 75(6): 456-470.

21. Salim S. Oxidative stress and the central nervous system. J Pharmacol Exp Ther (2017); 360: 201-205.

22. Rice D and Barone S Jr. Critical periods of vulnerability for the developing nervous system: evidence from humans and animal models. Environ Health Perspect (2000);108 (3): 511-533.

23. Carlson ES, Tkac I, Magid R, Connor MB, Andrews NC, Schallert T, Gunshin H, Georgieff MK and Petryk A. Iron is essential for neuron development and memory function in mouse hippocampus. J Nutr (2009); 139: 672-679.

24. Piloni NE, Reiteri M, Hernando MP, Cervino $\mathrm{CO}$ and Puntarulo S. Differential effect of acute iron overload on oxidative status and antiolxidant content in regions of rat brain. ToxicolPathol (2017); 45(8): 1067-1076.

25. Park J, Lee DG, Kim B, Park S-J, Kiim J-H, Lee $\mathrm{S}-\mathrm{R}$, Chang K-T, Lee H-S and Lee D-S. Iron overload triggers mitochondrial fragmentation via clcineurin sensitive signals in HT-22 hippocampal neuron cells. Toxicol (2015); 337: 39-46.

26. Healy S, McMahson J, Owens P and FitzGerald U. Significant glial alternations in response to iron loading in a novel organotypic hippocampal slice culture model Sci Report (2016); 6: 36410.

27. Fernandez LL, Carmona M, Potero-Otin M, Naudi A, Pamplona R, Schroder N and Ferrer I. Effects of increased iron intake during the neonatal period on the brain of adult A $\beta P P / P S 1$ transgenic mice. J Alzheimer's Dis (2010); 19: 1069-1080.

28. Desagher S and Martinou JC. Mitochondria as the central control point of apoptosis. Trends Cell Biol (2000); 10: 369-377.

29. GilleG andReichmannH. Iron-dependentfunctions of mitochondria-relation to neurodegeneration. J Neural Transm (2011); 118: 349-359.

30. Ma SM, Fang Z, Luo W, Yang $Y$, Wang C, Zhang Q, Wang $\mathrm{H}$, hen $\mathrm{H}$, Chan $\mathrm{CB}$ and Liu Z. The C-ETS2-TFEP axis promotes neuronal survival under oxidative stress by regulating lysosomal activity. Oxid Med Cell Longev(2016); 2016: ID 4693703, 16 pages 
31. Rohn TT. Caspase-cleaved TAR DNAbinding protein-43 is a major pathological finding in Alzheimer's disease. Brain Res (2008); 1228:189-198.

32. Pelizzoni I, Zacchetti D, Campanella A, Grohovaz $\mathrm{F}$ and Codazzi $\mathrm{F}$. Iron uptake in quiescent and inflammation activated astrocytes: A potentially neuroprotective control of iron burden. Biochimica et Biophysica Acta (2013); 1832: 1326-1333.

33. Amin SN, Younan SM, Youssef MF, Rashed LA and Mohamady I. A histological and functional study on hippocampal formation of normal and diabetic rats. F1000 Res (2013); 2: 151.

34. Abbott NJ, Patabendige AA, Dolman DE, Yusof SR and Begley DJ. Structure and function of the blood-brain barrier. Neurobiol Dis (2010); 37: 13-25.
35. McCarthy RC and Kosman DJ. Iron transport across the blood-brain barrier: development, neurovascular regulation and cerebral amyloid angiopathy. Cell Mol Life Sci (2015); 72: 709-27.

36. Won SM, Lee JH, Park UJ, Gwag J, Gwag BJ and Lee YB. Iron mediates endothelial cell damage and blood brain barrier opening in hippocampus after transient forebrain ischemia in rats. Exp Mol Med (2011); 43(2): 121-128.

37. Huang TT, Zou Y and Corniola R. Oxidative stress and adult neurogenesis - effects of radiation and superoxide dismutase deficiency. Semin Cell Dev Biol ((2012)); 23: 738-744.

38. Huang Y, Coupland NJ, Lebel M, Carter R, Seres P, Wilman AH and Malykhin NV. Structural changes in hippocampal subfields in major depressive disorders: A high field magnetic resonance imaging study. Biol Psych (2013); 74: 62-68. 
الملخص العزبى

مدى قابلية قرن آمون للسمية عقب تعرض ذكور الجرذان البيضاء الرضع لفرط عنصر الحديد بعد

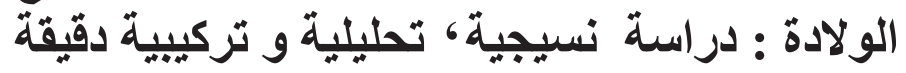

\section{أسماء فتحي داوود}

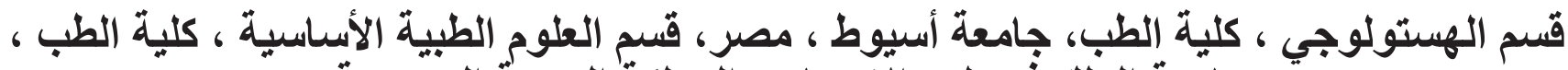

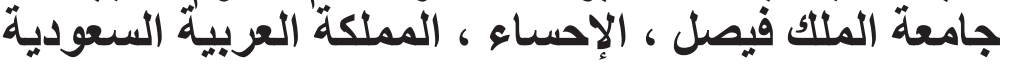

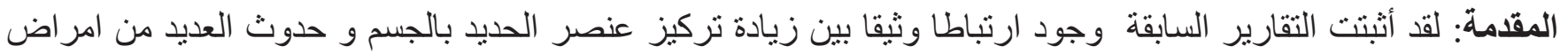

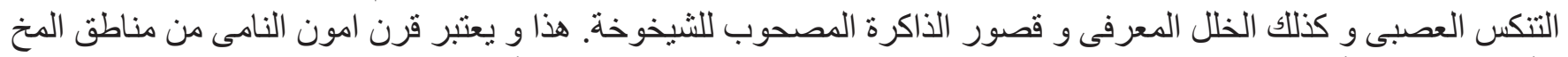

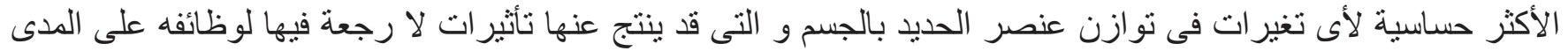
الطويل.

الهدف: تهدف هذه الدر اسة الى تقصى التغير ات المرضية المحتملة في مناطق قرن امون CA1 و CA3 و DG عقب تعرضها

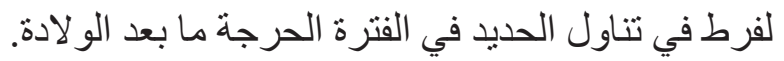

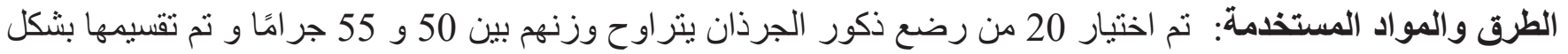

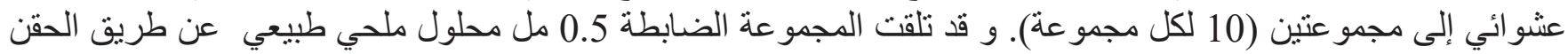

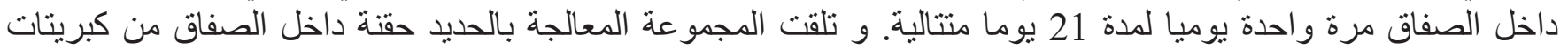

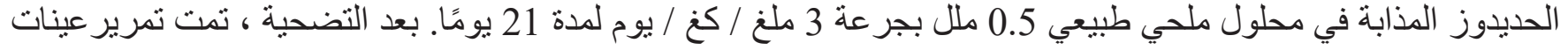
المخ للار اسة النسيجية، التحليلية ول التركيبية الديقية

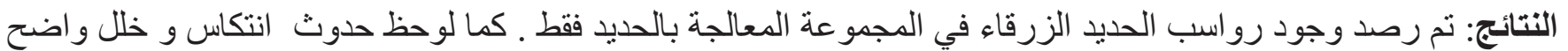

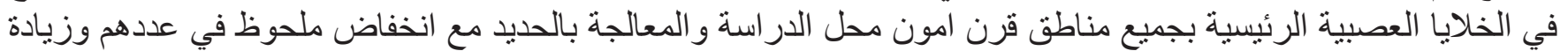

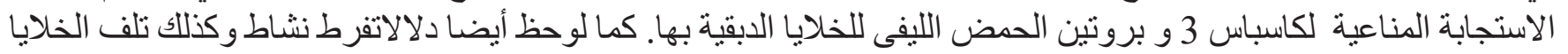

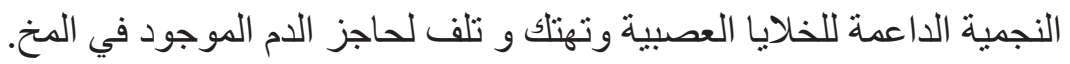

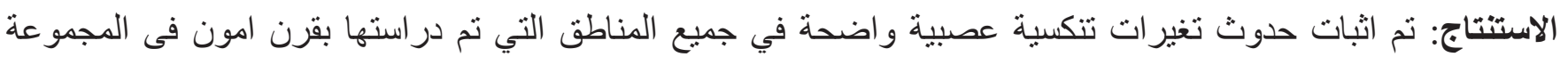

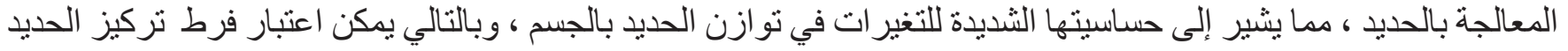
بالجسم من المسببات شديدة السمية على التركيب النسيجى لقرن امون النامى. 